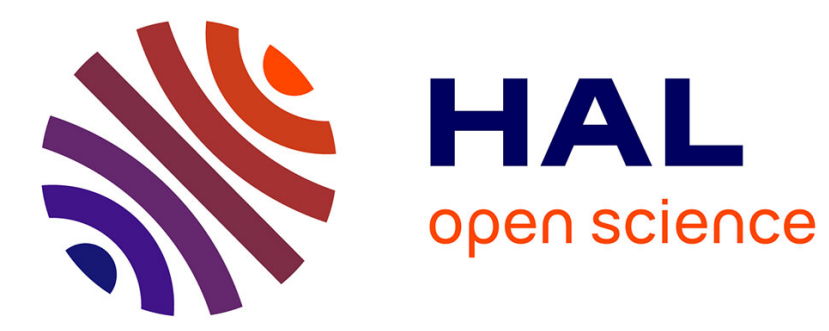

\title{
Muslims in France: identifying a discriminatory equilibrium
}

\author{
Claire L. Adida, David D. Laitin, Marie-Anne Valfort
}

\section{To cite this version:}

Claire L. Adida, David D. Laitin, Marie-Anne Valfort. Muslims in France: identifying a discriminatory equilibrium. Journal of Population Economics, 2014, 27 (4), pp.1039-1086. 10.1007/s00148-014-05121. halshs-00977076

\section{HAL Id: halshs-00977076 https://shs.hal.science/halshs-00977076}

Submitted on 10 Apr 2014

HAL is a multi-disciplinary open access archive for the deposit and dissemination of scientific research documents, whether they are published or not. The documents may come from teaching and research institutions in France or abroad, or from public or private research centers.
L'archive ouverte pluridisciplinaire $\mathbf{H A L}$, est destinée au dépôt et à la diffusion de documents scientifiques de niveau recherche, publiés ou non, émanant des établissements d'enseignement et de recherche français ou étrangers, des laboratoires publics ou privés. 


\title{
Muslims in France:
}

\section{Identifying a discriminatory equilibrium.*}

\author{
Claire L. Adida ${ }^{\dagger} \quad$ David D. Laitin ${ }^{\ddagger} \quad$ Marie-Anne Valfort $^{\S}$
}

July 3, 2013

\begin{abstract}
We analyze the assimilation patterns of Muslim immigrants in Western countries with a unique identification strategy. Survey and experimental data collected in France in 2009 suggest that Muslims and rooted French are locked in a sub-optimal equilibrium whereby (i) rooted French exhibit taste-based discrimination against those they are able to identify as Muslims and (ii) Muslims perceive French institutions as systematically discriminatory against them. This equilibrium is sustained because Muslims, perceiving discrimination as institutionalized, are reluctant to assimilate and rooted French, who are able to identify Muslims as such due to their lower assimilation, reveal their distaste for Muslims.
\end{abstract}

Keywords: Assimilation, Muslim and Christian immigrants, Discrimination, France. JEL: D03, J15, J71.

*The research reported in this paper is not the result of any for-pay consulting relationship. It was funded by the National Science Foundation, "Muslim Integration into EU Societies: Comparative Perspectives", Grant SES-0819635, David Laitin, P.I. We thank Ben Adida for building the web-based game for the March 2010 experiments. We also thank two anonymous referees, Yann Algan, John Bowen, Pierre Cahuc, Rafaela Dancygier, Henry Farrell, Harvey Feigenbaum, James Vreeland, and seminar/conference participants at the Georgetown University Center for Contemporary Arab Studies, Council of European Studies in Boston, and George Washington University Comparative Workshop for their very helpful comments.

${ }^{\dagger}$ University of California, San Diego. Department of Political Science. Social Sciences Building 301.9500 Gilman Drive, $¥ 0521$. La Jolla, CA 92093-0521. USA. E-mail: cadida@ucsd.edu.

${ }^{\ddagger}$ Stanford University. Department of Political Science. 100 Encina Hall. Stanford, CA 94305-6044. USA. E-mail: dlaitin@stanford.edu.

${ }^{\S}$ Corresponding author. Paris School of Economics - Paris 1 Panthéon Sorbonne University. 106-112, Boulevard de l'Hôpital. 75013 Paris. France. E-mail: marie-anne.valfort@univ-paris1.fr. Phone: 33(0)6 40 128407. 


\section{Introduction}

Debate rages in European societies as to whether Muslim immigrants can ever integrate (Caldwell (2009)). The Islamicist-inspired bombings in Spain (2004) and London (2005); the murder of Theo van Gogh in the Netherlands in 2004 for his depiction of Muslim sexuality; the riots in heavily-Muslim ghettoes of Paris (2005) and Stockholm (2013); the unceasing attacks on the Dane Kurt Westergaard for his unflattering portrayal of the Muslim prophet in a cartoon published in 2006; the legislative actions designed to ban Islamic dress in public places (in Belgium (2010), France (2010), and the Netherlands (2012)), and to prohibit Mosque construction in Switzerland (2009); the fervid reactions to the Muslim presence in Europe by radical fringe groups (that might have inspired the massacre in Norway in July 2011); and the violent attacks on soldiers in Britain and France by Islamist militants in 2013, all contribute to portray Muslims as posing a threat to Europe. One element of this threat is cultural, raising the question of whether Muslim immigrants and their descendants will assimilate into a common European culture. We therefore ask whether Muslims qua Muslims in Europe indeed show lower assimilation ${ }^{1}$ and, if so, what are the forces that sustain this deficit? These questions are urgent. Social cohesion in Europe is indeed at stake.

Evidence about the assimilation patterns of Muslim immigrants in Western countries is mixed. Using the United Kingdom (UK) Fourth National Survey of Ethnic Minorities, Bisin, Pattachini, Verdier and Zenou $(2008,2011 \mathrm{a})$ find that attachment to the culture of origin is higher for Muslims than for non-Muslims. For non-Muslims, this attachment attenuates with time spent in the UK; but for Muslims, attachment is unrelated to such time (see Dustmann (1996), Schields and Price(2002), Riphahn (2003), and van Ours and Veenman (2003) for similar conclusions). Yet, these results are at odds with those of Manning and Roy (2010) who, using the UK Labour Force Survey in 2001, analyze respondents' probability of answering "British" when asked to define their identity. They show that newly arrived immigrants almost never think of themselves as British and that no difference exists between Muslims and non-Muslims. Moreover, they find that the probability of reporting a British identity increases with the time spent in the UK, at a similar rate for Muslims and for non-Muslims (see Constant, Gataullina and Zimmermann (2009), Aleksynska (2011), and Georgiadis and Manning (2011) for similar results showing no Muslim effect).

These mixed results may partly derive from the difficulty of isolating a religion (Islam)

\footnotetext{
${ }^{1}$ Following Constant, Gataullina and Zimmermann (2009) or Gorinas (2013), we measure the degree of assimilation by the level of identification with ancestral homeland and with the host country.
} 
effect on assimilation patterns, due to possible confounds such as race, ethnicity or nationality. Indeed, Muslim immigrants typically migrate from Muslim countries, i.e. countries with few non-Muslim counterparts. This is the case, for example, of North African immigrants in France, Turkish immigrants in Germany and Pakistani and Bangladeshi immigrants in the UK. These confounds make it difficult to obtain a clean comparison for any European state, holding constant the country of origin, between the assimilation patterns of Muslim and non-Muslim immigrants.

The objective of this paper is twofold. First, we want to compare the assimilation patterns, on average and over time, of Muslim and non-Muslim immigrants without running into identification problems. We focus on France, which is home to the largest Muslim community in Western Europe both in absolute numbers (an estimated 4.7 million in 2010) and as a percentage of its population (an estimated $7.5 \%$ in 2010) according to the Pew Research Center (2011). To isolate a Muslim effect, we cannot study the principal Muslim immigrant group in France, viz. North Africans, as there is no matched set of North African Christians to whom North African Muslims could be compared. We focus instead on two ethno-linguistic groups originating in Senegal, the Joolas and the Serers. These ethnolinguistic groups are divided by religion, with one portion of them being Muslim and another portion being Christian. With the exception of religion, Senegalese Muslims (hereafter SM) and Senegalese Christians (hereafter SX) from these two ethno-linguistic groups are similar. They share the same culture and migrated to France during the same time (during the early 1970s), due to the same push factor (extensive drought conditions). Appendix A1 develops evidence that families from the Joola and Serer communities who converted to Christianity in the 19th century were not different from those who converted to Islam during the same period. Moreover, it shows that SM and SX individuals who were the first to migrate to France after WWII were similar across key characteristics: level of education and occupation in Senegal, as well as time of arrival to France.

To compare the assimilation patterns, on average and over time, of SM and SX, we rely on a survey that we conducted in 2009 among a set of second- and third-generation immigrants to France stemming from these two groups. Our results reveal that Muslim immigrants show significantly lower assimilation (i.e., higher attachment to their culture of origin and lower identification with the host culture and society) than do their Christian counterparts. Furthermore, we find that assimilation levels between SM and SX do not converge over the 
time elapsed since the arrival of the first migrant to France. ${ }^{2}$

What accounts for these results? Understanding the mechanism(s) behind the persistence of Muslims' lower assimilation in France constitutes the second objective of this paper. To do so, we rely on a field experiment ${ }^{3}$ we conducted in France in 2009. This experiment involves behavioral games that bring together SM and SX players with rooted French players (i.e., those citizens with four grandparents born inside metropolitan France) whom we refer to as FFF (i.e., French for at least three generations). Our games are designed to illuminate how SM and FFF behave toward each other when unconditional altruism and trust (i.e., belief about others' unconditional altruism) are at stake (as compared to the way SX and FFF behave toward each other). Our experiment reveals that SM are less cooperative (they show lower unconditional altruism and trust) - notably with rooted French players - than are SX, a result that is consistent with our survey finding that SM have assimilated less in France than have SX. Moreover, our games show that the persistence of Muslims' lower assimilation is consistent with Muslims and rooted French being locked in a sub-optimal equilibrium whereby: (i) rooted French exhibit taste-based discrimination, that is they show lower unconditional altruism (but not lower trust), toward those they are able to identify as Muslims (due to the fact that these Muslims maintain recognizable Muslim first names); (ii) Muslims perceive French institutions as systematically discriminatory against them (and therefore assimilate less, although Muslims' lower assimilation may not only be due to rooted French discrimination).

Our findings are consistent with research in identity economics showing that if the cost of assimilation is sufficiently high (possibly due to taste-based discrimination by the dominant group), it becomes rational for members of the minority to eschew assimilation (see Laitin (1995), Akerlof and Kranton (2000), Fang and Loury (2005), and Bisin, Pattachini, Verdier and Zenou (2011c)). Consistent with these findings is the evidence on assimilation behavior after an exogenous shock to everyday relations. For example, after the 9/11 attacks in the U.S., hostility toward Muslims by non-Muslim Americans increased, and - relatedly - Muslim identification with the U.S. decreased (Davila and Mora (2005) and Gould and Klor (2012)).

\footnotetext{
${ }^{2}$ For three of the eighteen items measuring assimilation, we even find that these patterns diverge: SM lower assimilation decreases, while SX higher assimilation increases with the time spent in France.

${ }^{3}$ Technically, and relying on the taxonomy created by Harrison and List (2004), we have conducted a "framed field experiment" since, as we shall describe, we rely on a nonstandard subject pool, and these subjects receive an information set from the real world (the names of their game partners) that they can use in their game participation. We also ran a follow-up experiment in 2010 that also relied on a nonstandard subject pool that received an information set from the real world. Henceforth, for economy of expression, we refer to both interventions as "field experiments".
} 
Note that taste-based discrimination by the dominant group could induce the adoption by the minority of an in-group norm of punishment of assimilators that further increases the cost of assimilation. In racial relations in the U.S., in-group policing in which initial assimilators are subject to the epithet of "acting white" deters other African-Americans from assimilating (see Fryer and Torelli (2010) for empirical evidence of this phenomenon and Fordham and Ogbu (1986), Fordham (1996), McWhorter (2000), Austen-Smith and Fryer (2005) and Eguia (2012) for its rational account).

The paper proceeds as follows. In Section 2, we introduce our survey data and the regression framework we use to analyze them. In Section 3, we present our survey results. Section 4 describes the field experiment. Section 5 presents our experimental results. Section 6 checks for robustness. Section 7 summarizes our major conclusions and discusses their policy implications for the assimilation of Muslim immigrants in France.

\section{Survey data and regression framework}

In this section, we present our survey, the questions we use to compare SM and SX assimilation patterns in France, and the regression framework that allows us to analyze this difference, on average and over time.

\subsection{The survey}

Our survey of 511 Serers and Joolas in France, both Christians and Muslims, was administered from April to June 2009 by the professional survey firm Conseils-Sondages-Analyses (CSA). The survey relied on a non-random sample of 332 respondents initially contacted by linking Serer and Joola surnames with cell numbers, ${ }^{4}$ and interviewed by telephone, and 179 respondents interviewed face-to-face through a chain referral sampling procedure.

The following four eligibility criteria were used: the respondent had to: (1) be 18 to 40 years of age, (2) be born in France, (3) have at least one parent or grandparent born outside of France, and (4) have at least one Serer or Joola-speaking grandparent.

Quotas were used to ensure better sample representativeness. Respondents represent $1.2 \%$ of the eligible population-base. We define as SM (SX) individuals who reported to be Muslim (Christian) and to belong to a family of Muslim (Christian) tradition. Overall, 466

\footnotetext{
${ }^{4}$ To avoid bias, we did not rely on landlines, as they would tap only the wealthiest, and least typical, of the immigrant population.
} 
respondents (91\% of the whole sample) are classified as either SM or SX - 339 (or 73\%) are classified as SM and $127(27 \%)$ as SX. ${ }^{5}$

\subsection{Measuring assimilation patterns}

We rely on two categories of questions to measure the assimilation patterns of SM and SX. The first category, meant to capture a respondent's strength of identification with her ancestry, measures attachment to the respondent's country (Senegal) or continent (Africa) of origin. The second category captures a respondent's strength of identification with a now-secularized French culture and society. ${ }^{6}$

Attachment to the country or continent of origin is based on nine questions capturing: (i) whether the respondent has ever been to Africa; (ii) whether the respondent sends remittances to Africa; (iii) the degree of sympathy the respondent has toward Senegalese living in Senegal; (iv) whether the grandparents of the respondent's best friend originate from Senegal or other African countries; (v) whether the association the respondent belongs to is related to Senegal or to Africa; (vi) whether the respondent believes she shares much in common with people of the same country of origin as her family; (vii) whether the respondent wants to be buried in Senegal or in Africa; (viii) whether the respondent owns a home in Africa; (ix) whether the respondent disapproves of a Senegalese student who chooses not to renounce a scholarship in a top French university to take care of a sick mother in Senegal.

Respondent identification with French culture and society is based on seven questions capturing: (i) the degree of sympathy the respondent has toward French people; (ii) whether the respondent believes that immigrants should do whatever possible to avoid conflict with the hosting society; (iii) whether the grandparents of the respondent's best friend originate from France; (iv) whether the respondent shows political preferences that can be positioned on a typical left-wing/right-wing scale $;^{7}(\mathrm{v})$ whether the respondent considers herself to share much in common with French people; (vi) whether the respondent wants to be buried in France; (vii) whether the respondent approves of a scenario whereby a man of Senegalese

\footnotetext{
${ }^{5}$ The sample remains disproportionately Muslim, and this is not surprising given that Senegal is more than 90\% Muslim. Our identification of two Senegalese ethnic groups with substantial numbers of Christians is key, given the predominance of Islam in Senegal.

${ }^{6}$ Assimilation into French culture can be measured in part by the reduction in the level in which religion infuses other aspects of life. Indeed, in the 2006 World Values Survey for France, 13\% say that religion is very important to their lives; by contrast, in the 2008 Afrobarometer survey for Senegal, $95.8 \%$ say that religion is very important to their lives. Relying on our definition of assimilation, those who claim that religion is less important to their lives are thus assimilating into French norms.

${ }^{7}$ Politics in Senegal do not follow a typical left-wing/right-wing scale the way they do in France. Instead, the most common political dimensions in Senegal are region, Sufi order and cousinage (see Smith (2010)).
} 
origin hides from his white son's friends so that they may believe the son is rooted French.

The strength of the respondent's identification with the host culture and society is also captured by the respondent's degree of secularization. This degree of secularization is based on six questions measuring: (i) the importance of god in the respondent's life; (ii) whether the respondent would disapprove of her child marrying a religious-other; (iii) the degree of sympathy the respondent has toward people sharing the same religion; (iv) whether the best friend of the respondent is of the same religion; (v) whether the association the respondent belongs to is related to religion; ${ }^{8}$ (vi) whether the respondent believes she shares much in common with people of the same religion.

\subsection{The regression framework}

To isolate whether SM and SX assimilation patterns differ on average, we estimate Equation (1):

$$
y=a+b . \mathrm{SM}+c . \text { Time }+\mathbf{d}^{\prime} \cdot \mathbf{X}+\epsilon,
$$

where $y$ is the respondent's answer to one of the questions above. The dummy SM is equal to 1 if the respondent is SM and to 0 if the respondent is SX. Therefore, coefficient $b$ captures the difference between the answer provided by SM respondents and the answer provided by SX respondents. The variable Time is continuous and captures the number of years elapsed since arrival of the first migrant in the respondent's family. We also control for $\mathbf{X}$, a vector of socioeconomic characteristics of the respondent. This vector contains information on characteristics that are either pre-treatment (i.e., they concern features of the household before migration of the first migrant to France) or exogenous. The pre-treatment control is the education of the first migrant. The exogenous controls are the gender and the age of the respondent. We do not include post-treatment (i.e., post-migration) controls since those would capture part of the "Muslim" effect and therefore bias the coefficient of the dummy SM.

To compare the assimilation patterns of SM and SX over time, we estimate Equation (2):

$$
y=a+b . \mathrm{SM}+c . \mathrm{SM} . \text { Time }+d . \text { Time }+e . \text { SM.Age }+\mathbf{f}^{\prime} . \mathbf{X}+\epsilon .
$$

The variables SM, Time and $\mathbf{X}$ are defined as in Equation (1). Coefficient $d$ captures the

\footnotetext{
${ }^{8}$ Note that there is no difference in the probability of participating in an association between SM and SX.
} 
impact of Time on SX assimilation. ${ }^{9}$ The sum of coefficients $c$ and $d$ captures the impact of Time on SM assimilation. ${ }^{10}$ Consequently, coefficient $c$ (the coefficient of the interaction term SM.Time) provides the difference between the evolution of SM and SX assimilation patterns over time.

Note that one expects a positive correlation between the age of the respondent and the number of years elapsed since arrival of the first migrant in the respondent's family. This intuition is confirmed by our data (this correlation is equal to 0.39 and significant at the $1 \%$ confidence level). Therefore, we also control in Equation (2) for the interaction term SM.Age where the variable Age represents the age of the respondent, in order to ensure that coefficient $c$ does not partially capture an age effect. ${ }^{11}$

\section{Survey results}

In this section, we show (by estimating Equation (1)) that SM and SX assimilation patterns differ on average, with SM assimilation into French culture and society significantly lower than SX assimilation into French culture and society. We then show (by estimating Equation (2)) that there is no convergence between SM and SX, namely that SM lower assimilation persists over time.

\subsection{Do SM and SX assimilation patterns differ on average?}

The fourth column in Tables 1, 2a and 2b provides results from our estimation of coefficient $b$ in Equation (1) on the three sets of questions measuring the assimilation patterns of migrants: attachment to Senegal and Africa (Table 1), identification with France (Table $2 \mathrm{a}$ ), and secularization (Table $2 \mathrm{~b}$ ). This coefficient captures the difference in assimilation between SM respondents and SX respondents.

The fourth column in Table 1 presents OLS estimates for the coefficient of the dummy $\mathrm{SM}$ in Equation (1) over the set of questions that capture the attachment of the respondent to Senegal and/or Africa. This coefficient is always positive. Moreover, it is almost always

\footnotetext{
${ }^{9}$ Alternatively, coefficient $d$ could capture the fact that the first migrants in SX families systematically differ with regard to assimilation, depending on when they arrived to France. We rule out this possibility in the robustness checks.

${ }^{10}$ Alternatively, the sum of coefficients $c$ and $d$ could capture the fact that the first migrants in SM families systematically differ with regard to assimilation, depending on when they arrived to France. We rule out this possibility in the robustness checks.

${ }^{11}$ We thank an anonymous referee for bringing this issue to our attention.
} 
significant at least at the $90 \%$ confidence level. The exceptions are displayed in rows 3 and 9, which capture respectively: the degree of sympathy the respondent has toward Senegalese living in Senegal; and whether the respondent disapproves of a Senegalese student who chooses not to renounce a scholarship in a top French university to take care of a sick mother in Senegal. Still, across seven of our nine indicators, our results clearly indicate that, on average, SM respondents show significantly stronger attachment to Senegal and Africa as compared to their Christian counterparts. ${ }^{12}$

The fourth column in table 2a presents OLS estimates for the coefficient of the dummy $\mathrm{SM}$ in Equation (1) over the set of questions that capture identification with the French culture and society, excluding secularization. This coefficient is always negative. Moreover, it is always significant at least at the 90\% confidence level. Our results clearly indicate that, on average, the identification of SM respondents with the host culture and society is significantly lower than the one observed among SX. ${ }^{13}$

The fourth column of table $2 \mathrm{~b}$ presents OLS estimates for the coefficient of the dummy $\mathrm{SM}$ in Equation (1) over the set of questions that capture secularization. ${ }^{14}$ This coefficient is always positive. Moreover, it is almost always significant at least at the $90 \%$ confidence level. The exceptions are displayed in rows 3 and 5, which capture - respectively - the degree of sympathy the respondent has toward people sharing the same religion, as well as whether the association the respondent belongs to is related to religion. Across four of our six indicators, our results clearly indicate that, on average, SM respondents display significantly less secularization as compared to their Christian counterparts. ${ }^{15}$ In sum, our results reveal that Muslim immigrants show significantly lower assimilation (i.e., higher attachment to their culture of origin and lower identification with the host culture and society, with notably - lower secularization) than do their Christian counterparts.

\footnotetext{
${ }^{12}$ Controlling for the variable Time, as well as for the respondent's socioeconomic characteristics, substantially reduces the number of observations due to missing data for these controls. However, the significance level for the dummy SM does not change if we impute missing data (results available upon request).

${ }^{13}$ The significance level for the dummy SM does not change if we impute missing data, with the exception of row 2 (whether the respondent believes immigrants should do whatever possible to avoid conflict with the hosting society) where the dummy SM loses significance (results available upon request).

${ }^{14}$ Given the coding of our measures of secularization, a positive coefficient indicates less secularization.

${ }^{15}$ The significance level for the dummy SM does not change if we impute missing data, with the exception of row 3 (the degree of sympathy of the respondent toward people sharing the same religion) where the dummy SM becomes significant (results available upon request).
} 


\subsection{How does the difference in assimilation patterns between SM and SX evolve over time?}

In Table 3, we estimate coefficient $c$ in Equation (2), that is, whether assimilation patterns of SM and SX change over time. We restrict our attention to assimilation outcomes that saw a significant SM effect in Tables 1 through 2b. Our results show that the coefficient of the interaction term SM.Time is almost never significant, suggesting that SM do not catch up with matched SX respondents over time. Moreover, in the three instances (out of eighteen dependent variables) where the coefficient of the interaction term SM.Time reaches statistical significance, the effect indicates divergence, not convergence, in SM and SX assimilation over time. We elaborate on these results below.

In Table 3, Panel A presents OLS estimates for coefficient $c$ in Equation (2) over the set of questions that capture respondent attachment to Senegal and Africa. This coefficient is not significant in column 1 and in columns 3 through 6, indicating that higher SM attachment and lower SX attachment to their country/continent of origin do not change over time. ${ }^{16}$ Note, however, that this coefficient is positive and significant in columns 2 and 7 (whether the respondent sends remittances and whether the respondent owns a home in Africa), indicating a divergence between SM and SX over time. More specifically, SM are more likely - while SX are less likely - to send remittances and to own a home in Africa over time.

In table 3, Panel B presents OLS estimates for coefficient $c$ in Equation (2) over the set of questions that capture identification with French host culture and society excluding secularization. This coefficient is never significant, indicating that SM lower identification and SX higher identification with French culture and society do not converge over time. ${ }^{17}$

In Table 3, Panel C presents OLS estimates for coefficient c in Equation (2) over the set of questions that capture secularization. This coefficient is not significant in columns 2 through 4, indicating that higher SM attachment and lower SX attachment to their religion-of-origin persist over time. Note that this coefficient is positive and significant in column 1, indicating a divergence between SM and SX over time regarding the importance they attach to god. More specifically, this importance increases over time for SM, while it decreases over time for SX. ${ }^{18}$ Overall, our analysis shows that SM and SX assimilation patterns do not converge,

\footnotetext{
${ }^{16}$ These results hold when we impute missing data, with the exception of column (6) where the positive coefficient of the interaction term SM.Time becomes significant (i.e., SM and SX attachment to Senegal and Africa diverges over time). More precisely, the desire to be buried in Senegal or in Africa increases over time for SM, while it decreases over time for SX (results available upon request).

${ }^{17}$ These results hold when we impute missing data (results available upon request).

${ }^{18}$ These results hold when we impute missing data (results available upon request).
} 
and in some cases diverge, over time.

Note that selection in the first migrant-type would lead us to measure only a biased causal effect of the duration of stay on SM and SX assimilation. More precisely, the first migrants in the families of our SM and SX respondents might systematically differ with regard to patterns of assimilation, depending on when they arrived to France. If so, then the persistent divergence in assimilation levels between SM and SX over time could be due not only to the impact of time spent in France, but also to differences in assimilation levels at the time of initial migration. We address this issue in the robustness checks and find no empirical support for selection among the first migrants.

\subsection{Discussion of our identification strategy}

Our results reveal that Muslim immigrants show significantly lower assimilation (i.e., higher attachment to their culture of origin and lower identification with the host culture and society, with - notably - lower secularization) than do their Christian counterparts. Moreover, they show that this difference does not decrease with the time immigrants spend in France. Given our identification strategy, ours is the first analysis to identify a specific Muslim effect on immigrant assimilation in France.

One may wonder however whether our results are not merely driven by differences that exist between SM and SX in Senegal, rather than by differences between SM and SX experiences in France. ${ }^{19}$ Notably, SX are a minority while SM are a majority in Senegal. Thus, it could be that SM are assimilating in France just fine, but that SX had such a difficult time in Senegal that their assimilation in France is superior to that of the SM. The success of Jews from Russia to the U.S. or Maronites from Lebanon to Europe is consistent with this interpretation, although the selection issue (in which oppressed minorities with ambition are more likely to emigrate than majority group members with similar ambition) prevents us from drawing any causal inference from these examples. Yet, continued religious harmony in Senegal (Mecham (2006)) as well as similar economic outcomes between SM and SX in Senegal before migration (see Appendix A1) suggests that SX are not an oppressed minority there.

Still, the simple fact of belonging to a minority in the country of origin may sharpen one's adaptation skills even if this minority is not oppressed, by forcing one to adapt to the majority culture. The ideal identification strategy would therefore have consisted in studying

\footnotetext{
${ }^{19}$ We thank an anonymous referee for bringing this issue to our attention.
} 
immigrants in France from a country in which the two religions are evenly matched. Yet, we have not found a sizeable immigrant community in France that fits this description, and which has been established for a sufficiently long time to allow us to study the evolution of its assimilation pattern. It is worthwhile noting however that Adida, Laitin and Valfort (2013) provide evidence suggesting that our results are not simply driven by a "minority effect". Relying on the European Social Survey, these authors compare the integration pattern in Western European countries of Muslim and Christian immigrants from Bosnia and Herzegovina, the only country worldwide were the share of Christians and Muslims is almost equal. ${ }^{20}$ Even in this context - where Christians are not a religious minority in their country of origin - Christians report significantly less discrimination in Western European host countries than do their Muslim counterparts.

\section{Experimental set up}

The second objective of this paper is to understand the mechanisms underlying the persistence of lower assimilation of Senegalese Muslims in France. To do so, we rely on field experiments that we conducted in France in 2009 (the same year we collected our survey data) and in 2010. The 2009 field experiment brings together rooted French (FFF), SM and SX in the context of behavioral games. In this section, we first present our 2009 subject pool. We then describe our 2009 games, designed to allow us to examine how SM and FFF (relative to SX and FFF) behave toward each other with regard to unconditional altruism and trust (i.e., belief about others' unconditional altruism). Finally, we explain, based on the 2010 field experiment, how we identify that FFF condition their behavior specifically on the Muslimness of our players, rather than on some other factor, i.e. the foreignness of players' names.

\subsection{The 2009 subject pool}

In March 2009, we set up a series of experimental games among FFF, SM and SX. For those games, we recruited 27 Senegalese players: 16 self-identified as Muslims (SM) and 11 as Christians (SX). ${ }^{21}$ We relied upon three separate networks to recruit these Senegalese

\footnotetext{
${ }^{20}$ According to the 2009 Report on International Religious Freedom by the US Department of State, $97 \%$ of the population in Bosnia and Herzegovina is either Christian or Muslim. Muslims stand for $46.5 \%$ of this population (while Christians account for $53.5 \%$ ).

${ }^{21}$ Our subjects are coded by religious self-identification or, when that information is missing, ascribed religious heritage based on the advice of an ethnographer with expertise on Senegalese culture, who served
} 
players. Two of the networks came from the ethnographers who were conducting family histories for our wider research project, and who were asked to recruit subjects by merely telling them they had heard about experiments with a chance to earn a lot of money. No mention was to be made about Senegalese specificity or religion. The third network came from a Senegalese night watchman (not from the Joola or Serer communities) who worked at a student dorm. He was given a quota for the SM and SX and paid for each recruit who showed up for registration and participated in the games. Here again, no mention was to be made about Senegalese specificity or religion. Table 4 presents the results of a difference of means test comparing the socioeconomic characteristics of our SM and SX participants. SM and SX do not differ on critical characteristics such as gender, age, education or household income. The only characteristic on which they differ is religiosity, with SX being significantly more religious than SM. This introduces a bias we treat in our regressions by controlling for the socioeconomic characteristics of SM and SX players and notably for their religiosity. ${ }^{22}$

It is important to emphasize that African Muslims are less spontaneously associated with Islam in the French collective imagination because they know little to no Arabic and interact indiscriminately with African Muslims and African non-Muslims (Diop (1988)). Any evidence of FFF discrimination against SM should thus be interpreted as a lower bound on the magnitude of FFF anti-Muslim discrimination: levels of discrimination against Maghrebis, the Muslims who are at the center of public debates about the role of Islam in France, would almost certainly be higher (had there been a way to identify a Muslim effect from a Maghrebi immigrant sample in France) than those we find for Senegalese Muslims.

To complement our game sessions, we also recruited 53 non-Senegalese players. The ethno-religious breakdown of these 53 non-Senegalese players was as follows: 29 players, among whom 21 were FFF, were of European background; 12 players were of sub-Saharan African background; and 12 players were of North African background.

We recruited these players in the ethnically diverse setting of the 19th district of Paris. ${ }^{23}$ For our experiments to be unbiased, we could not give players the impression that we wanted to know if they were conditioning their moves on the religious backgrounds of our Senegalese players, and therefore needed to conduct the experiments in a setting in which the Senegalese

on our research team.

${ }^{22}$ Note that this bias, if anything, runs against us finding FFF discrimination against SM since SM participants are more moderate in their religious practices.

${ }^{23}$ According to the 1999 French census, the percentage of individuals living in this district who are born in France is 63.5 (against 82.4 for all of Paris). A good picture of the diversity in the 19th district is offered in the French film "Entre les murs" ("The Class" in its English-language version) that received the Palme d'Or at the 2008 Cannes Film Festival. 
players would not appear to be exceptional. The 19th district, with its high levels of national, ethnic and religious diversity, offered a solution that worked: in the exit surveys for the experiments, only one subject speculated that religion had anything to do with the purposes of the games, ${ }^{24}$ and only one Senegalese player out of a total 27 verbally wondered if there was something odd about having other players in the room from his Senegalese language group.

We used a stratified (by population density) but not always fully random recruitment procedure centered on the 21 metro stations of the 19th district. In a fully random protocol, we assigned a weight to each metro station based on the density of the area in which it is located, with the higher density stations getting more cards in our random draw. Each recruitment team drew a metro station for each recruitment day, and then a number from 1 to 10 to determine which passer-by to invite as a game recruit. But because we wanted to ensure a large number of interactions between our SX/SM sample and FFF, we deviated from this protocol to assure ourselves a sufficient number of FFF players. When potential subjects who looked as if they were FFF walked by, recruiters were instructed to ignore the sequence of selection, and to ask them to participate in our experiment. Passers-by who were willing to hear our appeal were told that they could win up to 148 euros for about two and a half hours of game participation, ${ }^{25}$ games which were designed to investigate "how people from Ile-de-France [Parisian region] make decisions about money."

Turn-downs were about 30 percent, introducing some bias that likely leads to an overrepresentation of individuals favorable to diversity among our sample (relative to a random sample of game participants). Indeed, those individuals who agreed to participate in our experiments were told that they would interact with others from the Ile-de-France region, a region commonly known to be ethnically and religiously diverse. We can test this intuition for FFF players. To do so, we compare the average political ideology of our FFF sample to that of a random sample of rooted French from the 2009 European Social Survey ("ESS" henceforth). We use a question that measures where respondents stand on a left-wing/rightwing scale, capturing a tendency to support social change versus a tendency to preserve traditional values. One's position on a left wing-right wing scale therefore reveals, among other things, attitudes toward diversity. In order to obtain a comparable group of rooted French respondents in our experiment and in the ESS, we selected a sub-sample of ESS re-

\footnotetext{
${ }^{24}$ In the exit questionnaire, we asked: "Que pensez-vous que notre équipe aura appris sur vous à travers vos décisions aujourd'hui?" [What do you think our team will have learned about you from the decisions you made today?]

${ }^{25}$ This stands for roughly 8.5 times the hourly minimum wage in France in 2009.
} 
spondents who were born in France and whose parents were born in France. Unfortunately, the ESS does not provide information about the birthplace of the respondents' grandparents. We thus cannot exclude ESS respondents with one or more grandparents born abroad: our sample of rooted French respondents from the ESS is thus, if anything, more open to diversity than would be a sample of rooted French respondents with four grandparents born in metropolitan France (the definition of FFF for our experimental games). This bias thus runs against us finding any difference between our FFF players and the rooted French respondents in the ESS, since we hypothesize that the latter are more open to diversity than a random sample of FFF. Table 5 presents the results of a difference of means analysis between our FFF and the ESS rooted French. It shows that our FFF sample is, on average, more left-wing than the random sample of rooted French respondents in the 2009 ESS (significant at the $99 \%$ confidence level). These results are confirmed by an OLS analysis reported in Table 6. In this table, the variable "European Social Survey" takes the value 1 if the individual is a respondent in the 2009 ESS and 0 if she is a participant in our 2009 experiment. The coefficient for this variable is always positive and highly significant, whether one controls for the gender (column 2), age (column 3), education (column 4) or household income (column 5) of the individual. We therefore have confirmation that FFF participants in our 2009 experiments are more open to diversity compared to a representative sample of FFF in France that same year. As a consequence, our results suffer from a bias that again leads to an underestimation of anti-Muslim discrimination on the part of FFF.

\subsection{The 2009 games}

The 2009 field experiment comprised two phases: a registration phase, during which we collected demographic and behavioral data that we later used for the composition of the player-sets ${ }^{26}$ and a game phase, during which subjects played a series of experimental games. We supervised eight sessions of games of 10 players each held in a rented private language school in the 19th district in Paris, over the course of two weekends, on Friday evenings after work and on Sunday. Three of the sessions were all male; three of the sessions were all

\footnotetext{
${ }^{26}$ At registration, we collected demographic data from participants, potentially priming them about identity issues, and thereby biasing our results. This is unlikely, however, given that at least two weeks separated the registration and game phases. Moreover, as explained in the text, what we told the players about our games and where we held the sessions served to downplay any suggestion that religious identities had any role in our intervention. The success of this strategy was revealed in our exit questionnaires, which asked participants what they thought our team had learned about them throughout the games: only 1 respondent out of a total 80 mentioned religion.
} 
female; and two of the sessions were mixed gender.

When subjects arrived at a game session, they were given a code number. They were then told that since they would be interacting with strangers for the next few hours, interactions would be more personal if they wrote their first names on a label and pasted that label on their chests. All subjects complied without question or concern. The only information players had about each other was therefore their looks, their manners, their dress and their first names. None wore any clothes or jewelry revealing religious affiliation, with the exception of one non-Senegalese player, who wore a headscarf signaling a Muslim identity. After check-in was completed, they were brought to an open room with the other players, given magazines to read, and monitored in a way that discouraged any conversation or interaction.

\subsubsection{The sequencing of the games}

The session was comprised of five experimental games which were all designed to examine how SM and FFF interact with one another (relative to SX and FFF): (i) a simultaneous trust game; (ii) a speed chatting game; (iii) a voting game; (iv) a dictator game; and (v) a strategic dictator game. ${ }^{27}$

In the following we describe and present evidence from the first of those games, the simultaneous trust game, which was the only game to take place before the socialization of players with one another (via the speed-chatting game). We also briefly refer to the results stemming from the other games (described in Appendix A2) to show that they are consistent with those obtained from the simultaneous trust game.

Note that the sequencing of our games raises a potential concern with contamination effects of previous games on players' behavior during the subsequent games, especially if players learned about other players' game decisions once they were allowed to interact and communicate freely (during the speed-chatting game). Such contamination is highly unlikely however. During our initial presentation of the experiments to the players, we emphasized that all game decisions would remain anonymous and private at all times. Moreover, in our instructions for the speed chatting game, we stressed that players were to get to know - in

\footnotetext{
${ }^{27}$ Throughout the session, and out of sight from the players, monitors kept a full account of all answers and earnings for each player. At the end of the session, as players answered an exit survey, the winnings for each player were placed in sealed envelopes for them to take home. Full protocols (in French, but with English translations) are available upon request. Here we review only what is necessary for interpreting the results presented in the subsequent section. We take this opportunity to thank our six recruiters and monitors for their incredible hard work, intellectual contributions throughout, and dedication to the project: Mathieu Couttenier, Jacinto Cuvi Escobar, Karine Marazyan, Etienne Smith, Josselin Thuilliez and Séverine Toussaert.
} 
French "faire connaissance avec" - their speed-chatting partners, meaning that players were instructed to find out information about who their partners were outside of the lab, not what their partners did during the game. Finally, we instructed all players to keep notes of their speed-chatting conversations. In these notes, there is no evidence that game-behavior information was exchanged during the speed-chatting game.

\subsubsection{The simultaneous trust game}

The 2009 simultaneous trust game was the first game participants played in our experimental set-up. In this game, subjects sat quietly in a waiting room (they were supervised such that they could not communicate with one another), and were called to a table in pairs. For each pair, one player was assigned the role of "sender" and the other "receiver". The pairs were created to ensure that all FFF in a session played the simultaneous trust game twice with each Senegalese player in that session, once for each role. Sender was allotted 3 euros in her account, and could send any amount $\{0,1,2,3\}$ to receiver (an amount that was known by the players to be tripled) by marking this amount on a sheet that receiver would never see. Receiver simultaneously marked on a sheet the sender would never see what percentage $\{0,1 / 3,2 / 3,1\}^{28}$ of the amount received would be sent back to the sender. ${ }^{29}$ After each play, the senders and the receivers returned to the waiting room, not knowing if they would be called again, or in what role.

The amount sent by the sender in the trust game is commonly considered by experimental economists as a sign of trust that Cox (2004) defines as being "inherently a matter of the beliefs that one agent has about the behavior of another" (Cox (2004): 263). In other words, the amount sent by the sender in the trust game captures the sender's belief that the receiver will be generous to her. However, the amount sent by the sender has also been shown to partially capture unconditional altruism, that is the sender's willingness to be generous to

\footnotetext{
${ }^{28}$ In order to avoid the equal split option from becoming a focal point in this game (Schelling (1960)), we did not offer it.

${ }^{29}$ The novelty of our simultaneous trust game with respect to the original trust game introduced by Berg, Dickhaut and McCabe (1995) is in the simultaneity of the decisions made by the sender and by the receiver. We preferred the simultaneous trust game over the original trust game for several reasons. Our objective was to treat each trust game played by our subjects as a one-shot game in order to mimic everyday life random encounters between strangers. It was therefore critical to avoid any reputation effect that would have occurred if receivers learned how much particular senders had sent in previous games. This procedure also brings a touch of realism since most interactions in real life happen under incomplete information. In this respect, removing sequentiality in the decision process looks less artificial. Furthermore, since our protocol introduced a socialization phase after the simultaneous trust game, in which players would get to know each other, we did not want their conversations to be biased by knowledge of their partners' actions during the simultaneous trust game.
} 
the receiver irrespective of what the receiver does (Fershtman and Gneezy (2001), Camerer (2003), Cox (2004) and Ashraf, Bohnet and Piankov (2006)). ${ }^{30}$ As for the amount sent back by the receiver in the simultaneous trust game, it is most plausibly interpreted as a signal of unconditional altruism and/or belief-based reciprocal altruism (i.e., an altruistic behavior based on the receiver's belief that the sender is being kind to her, keeping in mind that the actions of the sender and of the receiver are simultaneous). ${ }^{31}$

Put differently, the simultaneous trust game offers the advantage of capturing the two common types of discrimination, taste-based and statistical discrimination, on the side of both the sender and the receiver. More precisely, differences in unconditional altruism by the sender and/or the receiver should reveal taste-based discrimination. According to Becker (1957), taste-based discrimination indeed results from the "special disutility" which a player attaches to contact with another player (see Arrow (1998)). By contrast, differences in trust on the side of the sender and/or differences in belief-based reciprocal altruism on the side of the receiver should reveal statistical discrimination. Following Arrow (1972), statistical discrimination would indeed result from the negative belief which a player holds about the ability of another player to be kind to her. ${ }^{32}$ Although our simultaneous trust game, in itself, cannot distinguish between taste-based versus statistical discrimination, we exploit information from our other games to identify which one is at stake in our players' decisions.

\subsection{Disentangling FFF response to SM Muslimness from FFF re- sponse to $\mathrm{SM}$ foreignness}

Since SX and SM players were indistinguishable to FFF players in terms of dress and appearance, the only observable signals differentiating our Senegalese players were their first names, which were written on a label pasted on their chests. The names of our SM and SX players are listed in Table 7. As should be apparent, all the SX had names that are from the Christian Bible (and which are common French names), but none of the Muslim players did. From the point of view of the FFF, therefore, the SX had French names. However, the SM all had foreign names, some of them sounding Muslim, others sounding more African. This

\footnotetext{
${ }^{30} \mathrm{~A}$ third possible confound of positive transfers by the sender in the trust game is risk aversion. Yet, risk aversion hasn't been shown to be a serious confounding factor. For instance, Eckel and Wilson (2004) do not find behavioral risk measures to be significantly correlated with the decision to send in the trust game. They conclude that "subjects do not see trust as a problem of risk" (Eckel and Wilson (2004): 464).

${ }^{31}$ See Trivers (1971) on the role that reciprocal altruism plays in cooperation.

${ }^{32}$ Originally, Arrow introduced statistical discrimination to account for employers discriminating racially when they believe that the unobserved determinants of performance are correlated with race, which is observable (Arrow (1998): 96).
} 
raises a question: did SM and SX first names adequately signal their religious affiliation, or might they have signaled something else, such as foreignness? It is critical to test whether FFF behavior toward SM is a response to SM Muslimness or to SM foreignness.

To distinguish between these two possibilities, we recruited 50 FFF players from the 19th district in 2010 to participate, among other things, in a field experiment asking subjects to guess the ethnic and religious affiliations of names. We contracted the professional survey firm CSA to recruit respondents with addresses in the 19th district, and they did so relying on the phone directory. The sample was non-random, since it depended on the agreement of individuals who were called to participate in the experiment, but through quotas on neighborhood (quartier), gender, and age we guarded against systematic bias with respect to critical observable characteristics. ${ }^{33}$ Respondents were invited to register if and only if they reported that all four grandparents were born within France's continental boundaries, thereby qualifying as rooted French. The average age of players was 40, ranging from 20 to 59. Twenty-seven were female, and twenty-three were male. The games took place in an office building in the nearby 18th district, and were administered by computer, in groups of five. Each player sat in front of a computer without interacting with any of the other four players who were all sitting in front of their monitors.

The 50 FFF participants in our 2010 experiments played a Names Game allowing us to address whether the FFF in our 2009 experiments were responding more to the religion of their fellow players' first names or to their foreignness. FFF players were presented, sequentially, a series of names among which the names of our 2009 SM and SX players. For purposes of expediency, a random half of FFF players were shown half of SM and SX players' first names; the other half were shown the rest. For each name, FFF players were asked to guess the religious heritage of the person in question (Christian, Muslim, Buddhist, Jewish, Hindu, Animist). Figure 1 illustrates a sample screenshot of the Names Game. The 2010 Names Game informs us that SX were unambiguously identified as Christians, consistent with the fact that they had names that are from the Christian Bible (and which are common French names). However, SM were not unambiguously characterized as Muslims. More precisely, the Names Game allowed us to compute, for each SM first name, the percentage

\footnotetext{
${ }^{33}$ Recall that we find that our sample of rooted French players in 2009 is more left-wing in political ideology than is the ESS sample of rooted French, suggesting that our sample is more open to diversity than the average rooted French. We do not have a 2010 ESS to conduct such an analysis for our 2010 sample of rooted French players. However, our 2010 sample of rooted French players was recruited from the same district as our 2009 sample, and was introduced to the games in the exact same manner. It is therefore likely that the direction of the bias in 2010 is the same as the one we found in 2009 .
} 
of FFF who identified it as a "Muslim" name. Based on this information, we created two subgroups among SM: SMM ("Senegalese Muslims with Muslim names") were those whose first names were guessed as "Muslim" by more than $50 \%$ of the FFF answering that question; SMA ("Senegalese Muslims with African names") were the remaining SM. Table 8 shows that only those with first names of Arabic origin were considered as Muslims (SMM). ${ }^{34}$ By contrast, the remaining SM (SMA) were considered as Christians or affiliated to more traditional religions like Animism. ${ }^{35}$

\section{Are SM and FFF locked in a sub-optimal equilib- rium?}

In the following, we first show that our games reveal a pattern - viz. SM are less cooperative than are SX throughout our experimental games - that is consistent with the lower assimilation levels we have identified for SM (relative to SX) in our survey. We then suggest that this lower assimilation (and its persistence, as documented in our survey data) is consistent with Muslims and rooted French being locked in a sub-optimal equilibrium whereby: (i) rooted French exhibit taste-based discrimination against those they are able to identify as Muslims (due to the fact that these Muslims keep recognizable Muslim first names instead of adopting French-sounding names); (ii) Muslims perceive French institutions as systematically discriminatory against them (and therefore assimilate less, many retaining Muslim first names). To be sure, Muslims' lower assimilation may not only be due to rooted French discrimination. More precisely, no active discrimination by rooted French is necessary to explain that, upon arrival to France, SM show lower assimilation than do SX. Due to their religion, SX are indeed culturally closer to a Christian-heritage host country like France than are SM. Assimilating in France should therefore be an easier process for SX than for SM. However, if this is the story, then convergence between SM and SX assimilation should occur over time. The fact that this is not the case suggests that an additional barrier, possibly the discrimination of SM by rooted French, is at stake.

\footnotetext{
${ }^{34}$ Only two of the eleven SM with first names of Arabic origin were not characterized as "Muslim" by a majority of FFF (these first names are Ibou and Sidy). Similarly, only one of the seven SM with first names not of Arabic origin were characterized as "Muslim" by a majority of FFF (this first name is Ndeye).

${ }^{35}$ And indeed, although the bulk of Serers and Joolas have converted to Islam or Christianity, many of them continue to follow traditional beliefs (see Berg and Ruth (2009)).
} 


\subsection{Experimental evidence of assimilation patterns among SM and SX}

Our survey results clearly indicate that SM are more attached to their country of origin and less attached to the host culture and society than are SX in France, this difference persisting over time. If our games are consistent with this pattern, we expect SM players to be less cooperative (i.e., to show lower unconditional altruism and trust) toward their game partners in France than are SX.

To test this implication, we use data from the simultaneous trust game to estimate Equation (3):

$$
y=a+b .(\mathrm{FFF} \rightarrow \mathrm{SM})+c \cdot(\mathrm{SX} \rightarrow \mathrm{All})+d \cdot(\mathrm{SM} \rightarrow \mathrm{All})+\mathbf{e}^{\prime} \cdot \mathbf{X}+\mathbf{f}^{\prime} \cdot \mathbf{Z}+\mathbf{g}^{\prime} \cdot \mathbf{\Pi}+\epsilon .
$$

The dependent variable $y$ refers to the amount sent by the sender to the receiver if we focus on the sender's behavior, and to the amount sent back by the receiver to the sender if we focus on the receiver's behavior. The dummy (FFF $\rightarrow \mathrm{SM})$ is equal to 1 if player 1 is FFF and player 2 is $\mathrm{SM}$ and to 0 otherwise. ${ }^{36}$ The dummy (SM $\rightarrow$ All) is equal to 1 if player 1 is SM and player 2 is any of the players with which player 1 was matched, and to 0 otherwise. The dummy (SX $\rightarrow$ All) is equal to 1 if player 1 is $\mathrm{SX}$ and player 2 is any of the players with which player 1 was matched, and to 0 otherwise. The reference category is the dummy $(\mathrm{FFF} \rightarrow \mathrm{SX})$ that is equal to 1 if player 1 is FFF and player 2 is SX and to 0 otherwise. Consequently, the difference between coefficients $d$ and $c$ captures the difference between the behavior of SM player 1 and the behavior of SX player 1 toward their game partners. Put differently, this difference allows us to test whether SM hold back in their donations as sender or receiver as compared to SX. Moreover, coefficient $b$ captures the difference between the behavior of FFF player 1 toward SM player 2 and the behavior of FFF player 1 toward SX player 2. We rely on this information when we tackle the next section dedicated to whether FFF players 1 discriminate against SM players 2 (as compared to SX players 2). Equation (3) also controls for the socioeconomic characteristics of players 1 and 2 (gender, age, household income, education, religiosity and whether the player knows players who participated in previous game sessions) that are denoted by $\mathbf{X}$ and $\mathbf{Z}$ respectively. Finally,

\footnotetext{
${ }^{36}$ Player 1 is the sender when we analyze the amount sent and the receiver when we analyze the amount sent back. Player 2 is the receiver when we analyze the amount sent and the sender when we analyze the amount sent back. In Equation (3), we not only focus on the behavior of SM and SX toward their game partners, but also on the behavior of FFF toward SM and SX. This is because we rely on Equation (3) in the following section to test whether FFF discriminate against SM in the simultaneous trust game.
} 
we introduce $\Pi$, a vector of session fixed effects. Note that standard errors are clustered at the player 1 level, since different game decisions by the same player 1 cannot be considered independent of one another.

That SM senders are significantly less cooperative toward all game partners is shown on Table 9. Columns 1, 3, 5 and 7 present OLS estimates from four model specifications of Equation (3), restricting our attention to the amount sent in the simultaneous trust game. In column 1, we control for the ethnic identity of the sender and of the receiver only. In column 3 , we add session fixed effects. In column 5, we include the socio-demographic characteristics of the sender and of the receiver. Since this inclusion generates a reduction in the sample size from 178 to 134 observations due to missing values for the income, education and religiosity of some of the senders and receivers, we run a multiple imputation analysis in column 7. The last Wald test at the bottom of Table 9 shows that the difference between coefficients $\mathrm{d}$ (in row (5)) and c (in row (4)) is significant in the most complete specifications presented in columns 5 and $7 .^{37}$

Results are the same, as revealed in Table 10 (columns 1, 3, 5 and 7), when we restrict our attention to the amount sent back in the simultaneous trust game. Here, with OLS estimates from four model specifications of Equation (3), controls are introduced stepwise, as in Table 9. Again, since the inclusion of the socio-demographic characteristics of the sender and receiver generates a reduction in the sample size from 166 to 129 observations due to missing values, we run a multiple imputation analysis in column 7 . The last Wald test at the bottom of Table 10 again shows that the difference between coefficients d (in row (5)) and c (in row (4)) is significant in the most complete specifications presented in columns 5 and 7, showing that SM receivers send back significantly less to their game partners than do SX receivers. In sum, the results from the 2009 simultaneous trust game, revealing that SM are outliers among our 19th district players, are consistent with those of our survey, which indicate that SM are less assimilated in France than are SX. It is important to emphasize that we observe the same pattern in the games that followed the socialization phase (via the speed-chatting game): SM allocate significantly less as leaders in a voting game (where elected group leaders distributed a 30-euro prize between themselves and their arbitrarilydesignated electors) than do SX, and SM give significantly less as donors in the subsequent dictator game than do SX (results available upon request).

\footnotetext{
${ }^{37}$ We ignore columns 2, 4, 6 and 8 for now, which are analyzed in Section 5.2.1
} 


\subsection{Do FFF discriminate against SM and why?}

In this section, we uncover one reason why SM assimilate less than do SX in France. Specifically, we investigate FFF discrimination against SM, and whether such discrimination is taste-based, statistical, or both.

\subsubsection{Do FFF discriminate against SM?}

We analyze the potential discriminatory behavior of our players in the simultaneous trust game by relying on Equation (3) and Equation (4) which introduces the distinction between SM with recognizable Muslim names (i.e., SMM) and SM without recognizable Muslim names (i.e., SMA):

$$
\begin{aligned}
y= & a+b \cdot(\mathrm{FFF} \rightarrow \mathrm{SMM})+c .(\mathrm{FFF} \rightarrow \mathrm{SMA}) \\
& +d \cdot(\mathrm{SX} \rightarrow \mathrm{All})+e \cdot(\mathrm{SM} \rightarrow \mathrm{All}) \\
& +\mathbf{f}^{\prime} \cdot \mathbf{X}+\mathbf{g}^{\prime} \cdot \mathbf{Z}+\mathbf{h}^{\prime} \cdot \mathbf{\Pi}+\epsilon .
\end{aligned}
$$

The dummy $(\mathrm{FFF} \rightarrow \mathrm{SMM})$ is equal to 1 if player 1 is FFF and player 2 is $\mathrm{SMM}$ and to 0 otherwise. The dummy (FFF $\rightarrow$ SMA) is equal to 1 if player 1 is FFF and player 2 is SMA and to 0 otherwise. The reference category is again the dummy (FFF $\rightarrow \mathrm{SX}$ ), equal to 1 if player 1 is FFF and player 2 is SX and to 0 otherwise. As a consequence, coefficient $b$ captures the difference between the behavior of FFF player 1 toward SMM player 2 and the behavior of FFF player 1 toward SX player 2. In other words, it tells us whether rooted French discriminate against Muslims with recognizable Muslim names relative to otherwisematched Christians. Coefficient $c$ captures the difference between the behavior of FFF player 1 toward SMA player 2 and the behavior of FFF player 1 toward SX player 2. In other words,

it tells us whether rooted French discriminate against Muslims without recognizable Muslim names relative to otherwise-matched Christians. Put differently, coefficients $b$ and $c$ allow us to test whether FFF player 1's discrimination against SM player 2 (if it exists) is a reaction to SM players 2's Muslimness or foreignness. Vectors $\mathbf{X}, \mathbf{Z}$ and $\mathbf{\Pi}$ are defined as in Equation (3).

Table 9 presents OLS estimates for Equation (3) (Models 1, 3, 5 and 7) and Equation (4) (Models 2, 4, 6 and 8), restricting our attention only to senders. The coefficient in row (1), columns 1, 3, 5, and 7 shows that the amount sent by FFF senders to SM receivers is lower than the amount sent by FFF senders to SX receivers, although never significantly so 
(see the p-value of the Wald test " $(1)=0$ " at the bottom of Table 9 ). The coefficients in rows (2) and (3), columns 2, 4, 6, and 8 allow us to extend this conclusion to the cases where we distinguish between SMM and SMA receivers (see the p-value of the Wald test " $(2)=0$ " and " $(3)=0 "$ at the bottom of Table 9$)$. The OLS analysis therefore cannot disconfirm the null hypothesis that FFF send equally to SX and SM, even those with recognizable Muslim names.

What happens when FFF act as receivers in the simultaneous trust game? Table 10 presents OLS estimates for Equation (3) (Models 1, 3, 5 and 7) and Equation (4) (Models 2, 4, 6 and 8), restricting our attention only to receivers. The coefficient in row (1), columns 1, 3, 5, and 7 shows that the amount sent back by FFF receivers to SM senders is lower (and significantly so in the most complete specifications) than the amount sent back by FFF receivers to SX senders. Moreover, the coefficients in rows (2) and (3), columns 2, 4, 6, and 8 reveal that the significantly lower amount sent back by FFF receivers to SM senders is driven by FFF discrimination against SMM, not by FFF discrimination against SMA (see the pvalue of the Wald test " $(2)=0 "$ and " $(3)=0$ " at the bottom of Table 10$)$. The OLS analysis therefore uncovers that FFF receivers discriminate against SM, and more precisely against SMM. It is important to stress that the non significant difference between the amounts sent back by FFF receivers to SMA and SX senders is not due to the low number of observations associated with those interactions. A difference of means analysis (not shown, but available upon request) indeed reveals that $\mathrm{FFF}$ receivers return exactly the same proportion of the amount they receive to SMA and SX senders (i.e., $48 \%$ on average). By contrast, FFF receivers return only an average $33 \%$ of the amount they receive to SMM senders.

Although the results from the 2009 simultaneous trust game reveal FFF anti-Muslim discrimination, it is important to emphasize that a different pattern is observed in the games following the socialization afforded by the speed-chatting game: FFF voters are not less likely to vote for a SM than for a SX leader, FFF leaders do not allocate less to SM than to SX voters, and FFF dictators do not give less to SM than to SX recipients (results available upon request). Put differently, we find that FFF a priori discriminate against SM (and more precisely against those they are able to identify as Muslims) but that they stop doing so after a basic conversation with their SM game partners. Note however that this finding no longer holds once the number of SM and SX in the game session increases. More precisely, Adida, Laitin and Valfort (2012b) show that, even after the socialization phase, FFF anti-Muslim discrimination is reactivated when the proportion of SM in the game session rises. The 
discrimination-reducing effect of socialization therefore appears particularly weak.

\subsubsection{What accounts for FFF anti-Muslim discrimination?}

Discrimination of FFF receivers against SM senders in the simultaneous trust game may reflect anti-Muslim taste-based discrimination, that is, lower unconditional altruism toward SM senders (as compared to SX senders). It may also reflect statistical discrimination, that is, lower reciprocal altruism toward SM senders due to the more pessimistic beliefs of FFF about SM senders' generosity toward them.

In our follow-up 2010 experiment among $50 \mathrm{FFF}$ players from the 19th district of Paris, we explicitly test the statistical discrimination hypothesis, i.e. whether FFF receivers are more pessimistic about SM senders' donation behavior toward players like them (FFF) in the 2009 simultaneous trust game. To do so, we analyze whether FFF hold different first-order beliefs about SM and SX, that is whether they are more pessimistic about SM versus SX unconditional altruism toward FFF. We also examine whether FFF hold different second-order beliefs about SM and SX, that is whether they expect SM beliefs about FFF unconditional altruism toward SM to be more pessimistic than SX beliefs about FFF unconditional altruism toward SX. The 2010 strategic dictator game, described in Appendix A3, allows us to test whether FFF hold different first-order beliefs about SM and about SX. Our results, also reported in Appendix A3, show that FFF guesses about SM unconditional altruism toward FFF recipients do not differ from FFF guesses about SX unconditional altruism toward FFF recipients. To test whether FFF hold different second-order beliefs about SM and SX, we rely on the 2010 double strategic dictator game. This game is described in Appendix A4. Our results, also reported in Appendix A4, show that FFF do not expect SM beliefs about FFF unconditional altruism toward SM to be more pessimistic than SX beliefs about FFF unconditional altruism toward SX. In other words, these results suggest that the difference between the amount sent back by FFF receiver to SM sender and the amount sent back by FFF receiver to SX sender reveals differences in FFF unconditional altruism toward SM and SX, not differences in FFF beliefs about SM and SX behavior.

An alternative interpretation of FFF discrimination of SM in the simultaneous trust game is that it reflects, not taste-based discrimination, but discrimination based on the belief that SX are poorer than SM and therefore need more money. In our 2010 experiment, we explicitly test whether FFF hold different beliefs about the income levels of our 2009 SM and SX players. Our test and results are reported in Appendix A5, and show that FFF 
expect no difference in the monthly income of SM and SX recipients. We can thus rule out this alternative interpretation.

Overall, our results show that FFF discriminate against Muslims they are able to identify as Muslims. ${ }^{38}$ Moreover, our experimental evidence suggests that this discrimination is tastebased. $^{39}$ Note that one may be surprised that rooted French mistakenly believe that SM and SX are equally cooperative. Indeed, their everyday real-world interactions with SM and SX should allow them to update their beliefs based on what they observe. However, this presupposes that FFF are actually interacting with SM: this would require us to believe that interactions between FFF and SM are sufficiently frequent to permit updating, an assumption belied by the levels of social isolation faced by most immigrant communities in France. ${ }^{40}$

\subsection{Do SM distrust their hosts more than do SX?}

We have shown that FFF discriminate against Muslims they identify as such. We now ask whether Muslims distrust their hosts, that is rooted French and French institutions, more than do SX (i.e., whether SM beliefs about rooted French and French institutions attitudes toward SM are more negative than SX beliefs about rooted French and French institutions attitudes toward SX). If so, their non-assimilation can be best understood as part of an equilibrium. To address this question, we turn to the 2009 strategic dictator game ${ }^{41}$ and then the CSA survey to measure SM (relative to SX) distrust toward rooted French and

\footnotetext{
${ }^{38}$ Our finding contradicts the claim by Jacquemet and Yannelis (2012) according to which anti-minority discrimination by the dominant group in Western countries does not reflect discrimination against any specific minority, but rather discrimination against all groups which sound foreign (what they call "ethnic homophily").

${ }^{39}$ The fact that FFF do not treat SM and SX differently when FFF are senders, but that they do discriminate against SM when FFF are receivers brings additional support to the fact that FFF exhibit taste-based, not statistical discrimination. Indeed, the amount sent in the trust game, whether simultaneous or not, is commonly considered by experimental economists as a signal of trust (hence the name given to this game). In this context, if FFF exhibit taste-based discrimination, it is not surprising that they do not discriminate against SM when they behave as senders but that they do discriminate when they behave as receivers. Besides belief-based reciprocal altruism, the amount sent back in the simultaneous trust game indeed captures unconditional altruism.

${ }^{40}$ See Algan, Hémet and Laitin (2012) who show that even in the HLM sector where immigrant communities and rooted French live close to each other, housing surveys show very low levels of social interaction between them.

${ }^{41}$ Note that relying on this game runs against us finding any difference between SM and SX beliefs about FFF behavior toward SM and SX respectively. As already mentioned, the 2009 strategic dictator game and its companion, the 2009 dictator game, took place after the speed-chatting game, once we can no longer identify anti-Muslim discrimination on the part of FFF. Yet, our results show that this phenomenon is not anticipated (or fully anticipated at least) by Muslims since the 2009 strategic dictator game reveals differences between SM and SX beliefs about FFF behavior toward SM and SX respectively.
} 
French institutions respectively.

\subsubsection{Do SM distrust rooted French more than do SX? Results from the 2009 strategic dictator game}

The strategic dictator game (see Appendix A2 for the protocol) helps us to determine the beliefs of our 2009 players about FFF unconditional altruism toward the various recipients in the 2009 dictator game. Namely, our analysis of this game - in which players are rewarded for correctly guessing the actual donations in the dictator game of an FFF player, to SM and SX faces - allows us to test whether (i) SM distrust FFF more than do SX, meaning that SM expectations about FFF unconditional altruism toward SM are significantly lower than SX expectations about FFF unconditional altruism toward SX; (ii) SM expect FFF to be less generous toward SM than toward SX (i.e., to discriminate against SM).

We test both hypotheses by estimating Equation (5) over a set of triads composed of SM or SX guessers, FFF dictators and SM or SX recipients:

$$
y=a+b .(\mathrm{SM} \rightsquigarrow \mathrm{FFF} \rightarrow \mathrm{SM})+c .(\mathrm{SM} \rightsquigarrow \mathrm{FFF} \rightarrow \mathrm{SX})+\mathbf{d}^{\prime} \cdot \mathbf{X}+e \cdot \text { Face }+\mathbf{f}^{\prime} . \boldsymbol{\Pi}+\epsilon
$$

where $y$ refers to the guesses about FFF dictators' donations. The dummy (SM $\rightsquigarrow$ FFF $\rightarrow$ $\mathrm{SM}$ ) is equal to 1 if the guesser is $\mathrm{SM}$ and the recipient is $\mathrm{SM}$ and to 0 otherwise. The dummy ( $\mathrm{SM} \rightsquigarrow \mathrm{FFF} \rightarrow \mathrm{SX}$ ) is equal to 1 if the guesser is $\mathrm{SM}$ and the recipient is $\mathrm{SX}$ and to 0 otherwise. The reference category is the dummy (SX $\rightsquigarrow$ FFF $\rightarrow$ SX) that is equal to 1 if the guesser is SX and the recipient is SX and to 0 otherwise. As a consequence, coefficient $b$ captures the difference between SM expectations about FFF dictators' donations to SM recipients and SX expectations about FFF dictators' donations to SX recipients. In other words, coefficient $b$ allows us to test whether SM distrust rooted French more than do SX (i.e., whether SM beliefs about FFF unconditional altruism toward SM is lower than SX beliefs about FFF unconditional altruism toward SX). Moreover, the difference between coefficient $b$ and coefficient $c$ captures the difference between SM expectations about FFF dictator donations to SM recipients and SM expectations about FFF dictator donations to SX recipients. In other words, this difference allows us to test whether SM expect FFF anti-Muslim discrimination, that is whether SM expect FFF to be less generous toward SM than toward SX. $\mathbf{X}$ is a vector of control for a series of socioeconomic characteristics of the guesser (gender, age, household income, education, religiosity and whether the player knows players who participated in previous game sessions). Additionally, in order to run a 
within-face analysis, we introduce the Face dummy that is equal to 1 if the recipient is the Senegalese woman (and 0 if the recipient is the Senegalese man). Finally, we introduce $\mathbf{\Pi}$, a vector of session fixed effects. Note that standard errors are clustered at the guesser level since guesses from the same guesser cannot be considered independent of one another.

Table 11 presents OLS estimates from four model specifications of Equation (5). In column 1, we control for the ethnic identity of the guesser and of the recipient only. In column 2, we add recipient face fixed effects as well as session fixed effects. In column 3, we include the socio-demographic characteristics of the guesser. Since this inclusion generates a reduction in the sample size from 41 to 34 observations due to missing values, we run a multiple imputation analysis in column 4.

The coefficient in row (1) in columns 1,2 , and 3 is negative and significant at least at the $90 \%$ confidence level, confirming that SM expectations about FFF unconditional altruism toward SM are significantly lower than SX expectations about FFF unconditional altruism toward SX. ${ }^{42}$ In other words, our results reveal that SM distrust FFF more than do SX. However, the last Wald test at the bottom of Table 11 (which is never significant in all four columns) shows that SM higher distrust toward FFF is not accompanied by SM expectations of FFF discrimination against them. More precisely, SM do not expect FFF to be less generous toward SM than toward SX. They simply expect, more than do SX, lower FFF unconditional altruism toward both SM and SX.

This last result is consistent with the lessons we were able to draw from ethnographic interviews for our project. These interviews were conducted in 2009, and covered the assimilation patterns of 19 SM and 22 SX in the greater Paris region (Ile-de-France), Lyon, Nantes, and Bordeaux. ${ }^{43}$ Although SM respondents were more likely to report a sense of being unwanted in France as compared to SX respondents, only one of the SM respondents considered religion as the reason for such discrimination. By contrast, race was highlighted by a majority of SM, as well as by a majority of SX, as the reason underlying discrimination in France.

\footnotetext{
${ }^{42}$ Although this result holds both in a difference of means analysis (not shown here) and in the first three columns of Table 11, it loses significance in column 4 when the multiple imputation analysis is run (but the p-value of 0.16 associated with the coefficient in row (1) is close to statistical significance). This weakening of the significance is likely due to the fact that multiple imputation typically generates high standard errors when the number of observations is low (Equation (6) is estimated on 41 observations only).

${ }^{43}$ The project employed two ethnographers to run these interviews: Etienne Smith (then a Ph.D. candidate in political science at Sciences-Po, who had conducted extensive field research in Senegal, and speaks Wolof, the lingua franca of Senegal, a language spoken by nearly all of our respondents) and Mahnaz Shirali (a Ph.D. in Sociology at the Ecole des Hautes Etudes en Sciences Sociales who has published extensively on Muslim youth and gender in France and Iran).
} 
One should note that the difficulty of SM to figure out on which ground they are discriminated against by FFF is easily understandable: as already emphasized when we motivated our identification strategy, research on discrimination itself has so far been unable to disentangle a religious effect from other possible confounds such as race. However, if SM do not manage to clearly identify the cause of FFF discrimination in face-to-face interactions, one expects them to show better ability in correctly updating their beliefs when FFF behavior is mediated by French institutions (labor market, schooling system, administration ... etc). Two non mutually exclusive explanations could account for this discrepancy in SM ability to detect FFF discrimination. First, FFF are likely to avoid interpersonal interactions with Muslims, precisely because, as we have shown, they attach disutility (distaste) to contact with Muslims: Muslims therefore lack opportunities to correctly update their beliefs. Second, FFF may be susceptible to acting in a socially desirable way in a context where their actions are observable (which is not the case in our field experiment, where all player decisions were anonymous and private). By contrast, FFF cannot avoid dealing with Muslims - and thus discriminating against them - through French institutions. This is all the more true since, behind the veil of institutions, FFF individual actions are not observable, and thus not prone to social desirability bias. The following section puts this intuition to test.

\subsubsection{Do SM distrust French institutions more than do SX? Results from the CSA survey}

Seven questions in the CSA survey allow us to capture the respondent's distrust toward French institutions, and specifically (i) the schooling system, (ii) the police, (iii) the parliament, (iv) the administration, (v) the judicial system, (vi) the trade unions and (vii) private firms. We analyze SM and SX answers to those questions by estimating Equation (1). Results are reported in Table 12. We observe that the coefficient of the dummy SM is always positive. Moreover, with the exception of column 2 (distrust toward the police), it is always significant at least at the $95 \%$ confidence level. These findings clearly indicate that distrust toward French institutions is significantly higher among SM than among SX respondents, despite the fact that survey questions are prone to elicit socially desirable answers. ${ }^{44}$ These results are fully consistent with those stemming from the 2009 strategic dictator game according to which SM distrust FFF more than do SX.

\footnotetext{
${ }^{44}$ The significance level for the dummy SM does not change if we impute missing data, with the exception of column (3) (i.e., distrust toward the parliament) and column (5) (i.e., distrust toward the judicial system) where the dummy SM loses significance (results available upon request).
} 
Does the CSA survey allow us to go one step further by showing that SM expect French institutions to be more discriminatory toward SM than toward SX (a result that did not emerge among the face-to-face interactions of the 2009 strategic dictator game)? Six questions in the CSA survey measure whether the respondent considers that the following French institutions treat individuals on an equal basis: the police, the immigration authorities, the prefecture, the judicial system, the schooling system, and the Pôle Emploi (the French national employment agency). Results are reported in Table 13. We observe that the coefficient of the dummy SM is always negative and significant: SM are less likely than SX to agree that these various French institutions treat individuals on an equal basis. ${ }^{45}$

Overall, our findings reveal that SM distrust their hosts (both rooted French and French institutions) more than do SX but this distrust, as our survey instrument suggests, is fully recognized as such only in the context of evaluating French institutions. Combined with our result that rooted French exhibit taste-based discrimination against those they are able to identify as Muslims, these findings are best summarized in terms of a discriminatory equilibrium between rooted French and Muslims: Muslims, who perceive discrimination as institutionalized, are reluctant to assimilate and rooted French, who are able to identify Muslims as such due to their lower assimilation, reveal their distaste for Muslims by acting less altruistically towards them.

\section{Robustness checks}

Three factors could challenge our results. First, in the CSA survey, the initial migrant to France may systematically differ in his or her assimilation potential, depending on when s/he arrived to France. If this is the case, then the conclusion that SM lower assimilation and SX higher assimilation follow parallel trends over time (they do not converge) may not only capture the impact of the time spent in France on SM and SX assimilation, but also the fact that the initial level of assimilation upon arrival to France differs across these two groups, depending on when the first migrants in those groups settled in France. To ensure that this bias is not at work, it is therefore necessary to confirm that the gap between the assimilation of the first migrants who arrived earlier and those who arrived later does not differ across SM and SX families. Second, one may worry that SM lower cooperation toward

\footnotetext{
${ }^{45}$ The significance level for the dummy SM does not change if we impute missing data, with the exception of column (1) (i.e., agreement with the fact that the police treats individuals on an equal basis) and column (2) (i.e., agreement with the fact that immigration authorities treat individuals on an equal basis) where the dummy SM loses significance (results available upon request).
} 
their game partners merely captures the fact that SM and SX are characterized by different gender norms. Adida, Laitin and Valfort (2012a) indeed show that SM men are significantly less generous toward women in general than are SX men, while SM women are significantly less generous toward women of their in-group than are SX women. We therefore need to test whether our results hold when we exclude cases where the dictator is male and the recipient is female, as well as cases where the dictator and the recipient are both SM or SX females. Third, our result that FFF receivers discriminate against SM senders in the simultaneous trust game may be biased by the influence of facial traits. In an original trust game, Eckel and Wilson (2006) indeed show that attractive receivers are viewed as more trustworthy: they are trusted at higher rates by the sender and as a consequence receive more from her. We must therefore test whether FFF anti-Muslim discrimination is robust to controls for the beauty, friendliness and trustworthiness FFF could infer from the facial traits of our 2009 players.

\subsection{Do the first migrants who arrived earlier and those who ar- rived later systematically differ in terms of assimilation across SM and SX families?}

To measure the assimilation of the first migrant to France, we focus on respondents' answers to three questions in the CSA survey: (i) whether the first male migrant married a non-French (African) woman; (ii) whether the first migrant married someone of the same religion; (iii) whether all the parents and grandparents of the respondent married someone of African background. In the following, we first investigate whether the results obtained based on these questions are consistent with our finding according to which SM show, on average, lower assimilation than do SX. We then tackle the critical issue of whether SM and SX first migrants systematically differ in terms of assimilation upon arrival in France, depending on when they arrived.

Table 14, models (1), (3) and (5) test Equation (1) applied to the three survey measures above. The positive and significant coefficient of the dummy SM in all three columns confirms that SM first migrants are significantly more attached to their continent of origin and significantly less secularized than are SX first migrants, holding their time of arrival at its average. ${ }^{46}$ These results are fully consistent with our finding according to which SM show,

\footnotetext{
${ }^{46}$ These results hold if we run a multiple imputation analysis.
} 
on average, lower assimilation than do SX.

Does the gap between the assimilation of the first migrants who arrived earlier and those who arrived later differ across SM and SX families? Table 14, models (2), (4) and (6) estimate Equation (2) applied to the three survey measures above. The coefficient of the interaction term SM.Time is never significant, indicating that the difference in assimilation of first migrants between SM and SX families does not depend on the time of arrival of these first migrants. ${ }^{47}$ Our main survey-based result according to which SM lower assimilation and SX higher assimilation follow parallel trends over time is therefore only due to the impact of the time spent in France, not to the additional impact of differences in assimilation between SM and SX first migrants that would depend on when they settled in France.

\subsection{Does lower SM cooperation toward their game partners sim- ply reflect SM gender norms?}

Adida, Laitin and Valfort (2012a) show that SM men are significantly less generous toward women in general than are SX men, while SM women are significantly less generous toward women of their in-group than are SX women. We therefore need to test whether SM lower cooperation toward their game partners hold when we exclude cases where the dictator is male and the recipient is female, as well as cases where the dictator and the recipient are both SM or SX females. Our results (not shown, but available upon request) confirm that SM cooperate significantly less in the 2009 simultaneous trust game (although we lose significance regarding the amount sent back), voting game and dictator game when such cases are excluded. Put differently, lower SM cooperation toward their game partners is not driven by the fact that SM and SX gender norms differ.

\subsection{Is FFF behavior in the simultaneous trust game robust to controlling for the facial traits of their game partners?}

To control for the influence of facial traits in the simultaneous trust game, we asked FFF participants in our 2010 experiments to examine pictures of our 2009 players and evaluate their beauty, friendliness and trustworthiness. More precisely, in this Beauty game, FFF players were shown, sequentially, a series of photographs of our Senegalese and FFF participants in

\footnotetext{
${ }^{47}$ These results hold if we run a multiple imputation analysis.
} 
our 2009 experiments. ${ }^{48}$ For each photograph, FFF players were asked to choose, for each of 3 pairs of adjectives (pretty/ugly; friendly/unfriendly; trustworthy/untrustworthy) which one best corresponded to the photographed player. Six options were available for each pair. For instance, for the pair (friendly/unfriendly), the player could select: "very friendly", "friendly", "somewhat friendly", "somewhat unfriendly", "unfriendly", "very unfriendly". Figure 2 illustrates a sample screenshot of the 2010 Beauty game.

We assign to each Senegalese and FFF players from our 2009 experiments the average beauty, friendliness and trustworthiness scores assigned to them by our $2010 \mathrm{FFF}$ players. We add these controls in our OLS estimation of Equation (3) and Equation (4) for the amount sent back by the receiver.

Table 15 reports the OLS estimates with imputation of missing values (note that the controls for the facial traits of the 2009 SM, SX and FFF players do not have any missing values). The significant negative coefficient of the dummy (FFF $\rightarrow \mathrm{SM})$ in column 1 confirms that FFF receivers send back significantly less to SM senders than to SX senders, even when we control for the facial traits of the sender and of the receiver. Moreover, the significant coefficient of the dummy (FFF $\rightarrow$ SMM) and the non significant coefficient of the dummy $(\mathrm{FFF} \rightarrow \mathrm{SMA})$ in column 2, indicate that this result holds only for SM recipients with recognizable Muslim names (SMM).

\section{Conclusion}

This paper relies on a unique identification strategy to compare the assimilation patterns, on average and over time, of Muslim and Christian immigrants in France and, furthermore, to identify the mechanism driving those differences. Survey-based results reveal that Muslim immigrants show significantly lower assimilation (i.e., higher attachment to their culture of origin and lower identification with the host culture and society) than do their Christian counterparts and that this difference persists regardless of the time immigrants spend in France. Experimental results indicate that the persistence of lower Muslim assimilation is consistent with Muslims and rooted French being locked in a sub-optimal equilibrium whereby: (i) rooted French exhibit taste-based discrimination against those they are able to identify as Muslims (due to the fact for instance that these Muslims keep recognizable Muslim first names instead of adopting French-sounding names) and (ii) Muslims perceive French

\footnotetext{
${ }^{48}$ For expediency, a random half of the $2010 \mathrm{FFF}$ players were shown half of our 2009 Senegalese and FFF players; the other random half were shown the other half of our 2009 Senegalese and FFF players.
} 
institutions as systematically discriminatory against them. This equilibrium is sustained because Muslims, perceiving discrimination as institutionalized, are reluctant to assimilate and rooted French, who are able to identify Muslims as Muslims due to their lower assimilation, reveal their distaste for Muslims by acting less altruistically toward them.

Our finding that rooted French and SM are locked in a sub-optimal equilibrium offers a potential explanation for the economic handicap faced by Muslims in France today ${ }^{49}$ and highlights the need for an equilibrium shift. Indeed, taste-based discrimination against Muslims has both direct and indirect negative effects on their prospects of economic success in France. With the direct effect, taste-based discrimination should lead Muslims to perform worse than their non-Muslim counterparts in markets that are critical for successful economic integration of immigrants, such as the labor, housing, or marriage markets. ${ }^{50}$ With the indirect effect, the discriminated minority assimilates less because of discrimination. This lower assimilation is expected to further strengthen the direct negative effect of anti-Muslim taste-based discrimination by potentially fueling rooted French statistical discrimination against Muslims. ${ }^{51}$

It is in the nature of equilibria that no party has an incentive to unilaterally change its behavior. This helps explain the ineffectiveness of many prejudice-reducing policies uncovered in Paluck and Green's extensive review of the literature (2009). What follows from our discussion is the necessity to take a two-pronged approach toward discrimination reduction. On the one hand, it would be useful to highlight to the rooted French that their behavior belies their republican ideals. How can this be done? Pope, Price and Wolfers (2011) have recently shown the benefits of broadcasting research findings on discrimination, especially in

\footnotetext{
${ }^{49}$ Adida, Laitin and Valfort (2010) identify that Senegalese Muslim households in France earn, on average, 400 euros less than their Christian counterparts each month (the equivalent of $14 \%$ of the average monthly household income for France in 2009). See Constant and Zimmermann (2008) and Bisin, Pattachini, Verdier and Zenou (2011b) for further evidence on the relationship between low assimilation and poor economic performance in Europe.

${ }^{50}$ Adida, Laitin and Valfort (2010) confirm that significant anti-Muslim discrimination prevails in the French labor market. They compare the rate of interview callbacks received by two French applicants of Senegalese background showing the same educational and work experience but differing on religion, with a similar experimental design as in Bertrand and Mullainathan (2004). They confirm that the Muslim applicant faces high prejudice in France in 2009: she is 2.5 times less likely to receive a callback for an interview than is her Christian counterpart.

${ }^{51}$ Our experimental results clearly show that rooted French players do not expect SM to be less cooperative than SX. However, rooted French more exposed to contact with minorities, like recruiters, may hold correct beliefs. Relying on 400 interviews with human resources managers in large French firms, Bouzar and Bouzar (2010) indeed show that H.R. personnel report partly conditioning their hiring decisions (this is at least a post facto rationalization of such decisions) on their beliefs about what Muslims will do to the esprit-de-corps of their work teams. Perception of Muslims' higher attachment to their religion and culture of origin is listed among the factors underlying their preferences for non-Muslims over Muslims.
} 
an institutional environment committed to fair play. These authors refer to the considerable media attention given to a working paper by Price and Wolfers in 2007 (but published in 2010) documenting that personal fouls are relatively more likely to be called against NBA basketball players when they are officiated by an opposite-race refereeing crew. Pope, Price and Wolfers (2011) show that this media coverage durably erased racial bias by referees. Their finding suggests that making public academic research on discrimination, like the one provided in this paper, could bring about meaningful change in religious discriminatory behavior, especially among the French, who aspire in their republican ideology to treat all citizens equally. On the other hand, Muslims can collectively reveal a commitment to assim-

ilate. One route would be to encourage them to do as many aspiring immigrant groups do around the world, that is, to give French-sounding first names to their children. While the rooted French (if distaste remains) are then likely to seek other clues for Muslim identity, this might serve the Muslim community well as a transitional tactic to induce an equilibrium shift. When it comes to social norms, equilibria shift are not trivial to induce (see Mackie (1996)); but this paper suggests that this is the challenge France faces in light of a harmful and potentially dangerous discriminatory equilibrium.

\section{References}

[1] Adida, Claire L., David D. Laitin, and Marie-Anne Valfort. 2010. "Identifying barriers to Muslim integration in France." Proceedings of the National Academy of Sciences, 107(52): 384-390.

[2] Adida, Claire L., David D. Laitin and Marie-Anne Valfort. 2012a. "Gender norms, Muslim immigrants, and economic integration in France." Unpublished manuscript.

[3] Adida, Claire L., David D. Laitin and Marie-Anne Valfort. 2012b. " "One Muslim is enough!" Evidence from a field experiment in France." Unpublished manuscript.

[4] Adida, Claire L., David D. Laitin and Marie-Anne Valfort. 2013. "Region of origin or religion? Understanding why immigrants from Muslim-majority countries are discriminated against in Western Europe." Unpublished manuscript.

[5] Akerlof, George A. and Rachel E. Kranton. 2000. "Economics and identity." Quarterly Journal of Economics, 115(3): 715-753. 
[6] Aleksynska, Mariya. 2011. "Civic participation of immigrants in Europe: assimilation, origin, and destination country effects." European Journal of Political Economy, 27(3): 566-585.

[7] Algan, Yann, Camille Hémet and David D. Laitin. 2012. "Diversity and local public goods: a natural experiment with exogenous residential allocation." Unpublished manuscript.

[8] Arrow, Kenneth J. 1972. "Some mathematical models of race discrimination in the labor market", in A.H. Pascal, ed. Racial discrimination in economic life, Lexington, Mass.: D.C. Heath, 187-204.

[9] Arrow, Kenneth J. 1998. "What has Economics to say About Racial Discrimination?" Journal of Economic Perspectives, 12(2): 91-100.

[10] Asante, Molefi Kete. 2009. "Serer." in M.K. Asante and Ama Mazama (eds), Encyclopedia of African Religion, Thousand Oaks, CA: Sage.

[11] Ashraf, Nava, Iris Bohnet and Nikita Piankov. 2006. "Decomposing trust and trustworthiness." Experimental Economics, 9(3): 193-208.

[12] Austen-Smith, David and Roland G. Fryer. 2005. "An economic analysis of "Acting White"." Quarterly Journal of Economics, 120(2): 551-583.

[13] Axelrod, Robert. 1984. The evolution of cooperation. Basic Books.

[14] Becker, Gary. 1957. The economics of discrimination. Chicago: University of Chicago Press.

[15] Berg, Elizabeth L. and Wan Ruth. 2009. Cultures of the World: Senegal. Benchmark Books: New York.

[16] Berg, Joyce, John Dickhaut, and Kevin McCabe. 1995. "Trust, Reciprocity and Social History." Games and Economic Behavior, 10(1): 122-142.

[17] Bertrand, Marianne and Sendhil Mullainathan. 2004. "Are Emily and Greg more employable than Lakisha and Jamal?" American Economic Review, 94(4): 991-1013. 
[18] Bisin, Alberto, Eleonora Patacchini, Thierry A. Verdier and Yves Zenou. 2008. "Are Muslim immigrants different in terms of cultural integration?" Journal of the European Economic Association, 6(2-3): 445-456.

[19] Bisin, Alberto, Eleonora Patacchini, Thierry A. Verdier and Yves Zenou. 2011a. "Errata corrige "Are Muslim immigrants different in terms of cultural integration?" Journal of the European Economic Association, 9(5): 1012-1019.

[20] Bisin, Alberto, Eleonora Patacchini, Thierry A. Verdier and Yves Zenou. 2011b. "Immigrants and the labour market." Economic Policy, 26(65): 57-92.

[21] Bisin, Alberto, Eleonora Patacchini, Thierry A. Verdier and Yves Zenou. 2011c. "Formation and persistence of oppositional identities." European Economic Review, 55(8): 1046-1071.

[22] Bouzar, Dounia and Lylia Bouzar. 2009. Allah a-t-il sa place dans l'entreprise? Albin Michel.

[23] Caldwell, Christopher. 2009. Reflections on the revolution in Europe: Immigration, Islam, and the West. New York: Doubleday.

[24] Camerer, Colin F. 2003. Behavioral game theory. Princeton: Princeton University Press

[25] Cannot-Brown, Willie. 2009. "Jola." in M.K. Asante and Ama Mazama (eds), Encyclopedia of African Religion, Thousand Oaks, CA: Sage.

[26] Constant, Amelie F. and Klaus F. Zimmermann. 2008. "Measuring ethnic identity and its impact on economic behavior." Journal of the European Economic Association, 6 (2-3): 424-433.

[27] Constant, Amelie F., Liliya Gataullina and Klaus F. Zimmermann. 2009. "Ethnosizing immigrants." Journal of Economic Behavior and Organization, 69(3): 274-287.

[28] Cox, James C. 2004. "How to identify trust and reciprocity." Games and Economic Behavior, 46(2): 260-281.

[29] Davila, Alberto and Marie Mora. 2005. "Changes in the earnings of Arab men in the US between 2000 and 2002." Journal of Population Economics 18(4): 587-601. 
[30] Dustmann, Christian. 1996. "The social assimilation of immigrants." Journal of Population Economics 9(1): 37-54.

[31] Diop, A.M. 1988. "Stéréotypes et stratégies", in R. Leveau and G. Kepel, ed. Les Musulmans dans la société française, Presses de la Foundation Nationale des Sciences Politiques, Paris, 77-98.

[32] Eckel, Catherine C. and Rick K. Wilson. 2004. "Is trust a risky decision?" Journal of Economic Behavior and Organization, 55(4): 447-465.

[33] Eckel, Catherine C. and Rick K. Wilson. 2006. "Judging a book by its cover: beauty and expectations in the trust game." Political Research Quarterly, 59(2): 189-202.

[34] Eguia, Jon X. 2012. "Discrimination and assimilation." Unpublished manuscript.

[35] Entre les Murs. 2008. Film directed by Laurent Cantet.

[36] Fang, Hanming and Glenn C. Loury. 2005. "Dysfunctional identities"” can be rational." American Economic Review, 95(2): 104-111.

[37] Fershtman, Chaim and Uri Gneezy. 2001. "Discrimination in a segmented society: an experimental approach." Quarterly Journal of Economics, 116(1): 351-377.

[38] Fordham, Signithia and John U. Ogbu. 1986. "Black students' school successes: coping with the burden of "Acting White"”. Urban Review, 18(3): 176-206.

[39] Fordham, Signithia. 1996. Blacked out: dilemmas of race, identity and the success at capital high. University of Chicago Press.

[40] Fryer, Roland G. 2009. "An empirical analysis of "Acting White"." Journal of Public Economics, 94(56): 380-396.

[41] Gastellu, Jean-Marc. 1981. L'égalitarisme économique des Serer du Sénégal. Paris: ORSTOM.

[42] Georgiadis, Andreas and Alan Manning. 2011. "Change and continuity among minority communities in Britain." Journal of Population Economics 24(2): 541-568.

[43] Gorinas, Cédric. 2013. "Ethnic identity, majority norms, and the nativeimmigrant employment gap." Journal of Population Economics, forthcoming. 
[44] Gould, Eric D. and Esteban F. Klor. 2012. "The long-run effect of 9/11: terrorism, backlash, and assimilation of Muslim immigrants in the West." Unpublished manuscript.

[45] Harrison, Glenn W and John A. List. 2004. "Field experiments." Journal of Economic Literature, 42(4): 1009-1055.

[46] Hertwig, Ralph and Andreas Ortmann. 2001. "Experimental practices in economics: a methodological challenge for psychologists?" Behavioral and Brain Sciences 24(3): 383-403.

[47] Jacquemet, Nicolas and Constantine Yannelis. 2012. "Indiscriminate discrimination: a correspondence test for ethnic homophily in the Chicago Labor Market." Labour Economics 19(6): 824-832.

[48] Jamison, Julian, Dean Karlan and Laura Schechter. 2008. "To deceive or not to deceive: the effect of deception on behavior in future laboratory experiments." Journal of Economic Behavior and Organization 68(3-4): 477-488.

[49] Kahneman, Daniel, Jack L. Knetsch, and Richard H. Thaler. 1986. "Fairness and the assumptions of economics." Journal of Business, 59(4): 285-300.

[50] Laitin, David D. 1995. "Marginality: a microperspective." Rationality and Society, 7(1): 31-57.

[51] Linares, Olga. 1992. Power, Prayer and Production: The Jola of Casamance, Senegal. Cambridge: Cambridge University Press.

[52] Mackie, Gerry. 1996. "Ending footbinding and infibulation: a convention account." American Sociological Review 61(6): 999-1017.

[53] Manning, Alan and Sanchari Roy. 2010. "Culture clash or culture club? National identity in Britain." Economic Journal, 120(542): F72-F100.

[54] McWhorter, John H. 2000. Losing the race: self-sabotage in black America. Free Press.

[55] Mecham, Quinn. 2006. From the sacred to the state: institutional origins of Islamist political mobilization. Ph.D. Thesis, Stanford University, Department of Political Science. Under review at Cambridge University Press. 
[56] Paluck, Elizabeth Levy and Donald P. Green. 2009. "Prejudice reduction: what works? A critical look at evidence from the field and the laboratory." Annual Review of Psychology, 60: 339-367.

[57] Pew Research Center Report. 2011. "The future of the global Muslim population. Projections for 2010-2030."

[58] Pope, Devin, Joseph Price and Justin Wolfers. 2011. "Awareness reduces racial bias." Unpublished manuscript.

[59] Price, Joseph and Justin Wolfers. 2010. "Racial discrimination among NBA referees." Quarterly Journal of Economics, 125(4): 1859-1887.

[60] Riphahn, Regina T. 2003. "Cohort effects in the educational attainment of second generation immigrants in Germany: an analysis of census data." Journal of Population Economics 16(4): 711-737.

[61] Schelling, Thomas C. 1960. The strategy of conflict. Cambridge, Massachusetts: Harvard University Press.

[62] Schields, Michael A. and Stephen Wheatley Price. 2002. "The English language fluency and occupational success of ethnic minority immigrant men living in English metropolitan areas." Journal of Population Economics 15(1): 137-160.

[63] Smith, Etienne. 2010. "Des arts de faire société: parentés à plaisanteries et constructions identitaires en Afrique de l'Ouest (Sénégal)." Doctorat de Science Politique, Institut d'Etudes Politiques de Paris.

[64] Trivers, Robert L. 1971. "The evolution of reciprocal altruism." Quarterly Review of Biology, 46(1): 35-57.

[65] van Ours, Jan C. and Justus Veenman. 2003. "The educational attainment of secondgeneration immigrants in The Netherlands." Journal of Population Economics 16(4): 739-753. 
Tables and Figures

Table 1: Comparing average SM/SX attachment to the country/continent of origin.

\begin{tabular}{|c|c|c|c|c|}
\hline & $\begin{array}{l}\text { SX } \\
\text { (a) }\end{array}$ & $\begin{array}{l}\mathrm{SM} \\
(\mathrm{b})\end{array}$ & $\begin{array}{l}\text { Raw diff. } \\
\text { (b)-(a) }\end{array}$ & $\begin{array}{l}\text { Regression-adjusted diff. } \\
\text { (b)-(a) }\end{array}$ \\
\hline (1) Visit: $=1$ if the respondent has ever been to Africa, $=0$ otherwise & $\begin{array}{c}0.850 \\
(\mathrm{~N}=127)\end{array}$ & $\begin{array}{c}0.920 \\
(\mathrm{~N}=339)\end{array}$ & $\begin{array}{c}0.070^{* *} \\
(\mathrm{~N}=466)\end{array}$ & $\begin{array}{l}0.105^{* *} \\
(\mathrm{~N}=295)\end{array}$ \\
\hline (2) Remit: $=1$ if the respondent sends remittances to Africa, $=0$ otherwise & $\begin{array}{c}0.520 \\
(\mathrm{~N}=127)\end{array}$ & $\begin{array}{c}0.584 \\
(\mathrm{~N}=339)\end{array}$ & $\begin{array}{c}0.064 \\
(\mathrm{~N}=466)\end{array}$ & $\begin{array}{l}0.102^{*} \\
(\mathrm{~N}=295)\end{array}$ \\
\hline (3) Sympathy: sympathy of the respondent toward Senegalese living in Senegal (from 1 to 10) & $\begin{array}{c}8.268 \\
(\mathrm{~N}=127)\end{array}$ & $\begin{array}{c}8.298 \\
(\mathrm{~N}=329)\end{array}$ & $\begin{array}{c}0.030 \\
(\mathrm{~N}=456)\end{array}$ & $\begin{array}{c}0.048 \\
(\mathrm{~N}=292)\end{array}$ \\
\hline $\begin{array}{l}\text { (4) Best friend: }=1 \text { if the grandparents of the respondent's best friend originate from Africa, ... } \\
\ldots=0 \text { otherwise }\end{array}$ & $\begin{array}{c}0.504 \\
(\mathrm{~N}=127)\end{array}$ & $\begin{array}{c}0.634 \\
(\mathrm{~N}=328)\end{array}$ & $\begin{array}{l}0.130^{* *} \\
(\mathrm{~N}=455)\end{array}$ & $\begin{array}{l}0.188^{* * *} \\
(\mathrm{~N}=290)\end{array}$ \\
\hline (5) Association: $=1$ if the association the respondent belongs to is related to Africa, $=0$ otherwise & $\begin{array}{c}0.286 \\
(\mathrm{~N}=42)\end{array}$ & $\begin{array}{c}0.425 \\
(\mathrm{~N}=134)\end{array}$ & $\begin{array}{l}0.139^{*} \\
(\mathrm{~N}=176)\end{array}$ & $\begin{array}{l}0.265^{* * *} \\
(\mathrm{~N}=127)\end{array}$ \\
\hline $\begin{array}{l}\text { (6) Commonalities: commonalities the respondent believes she shares with people from... } \\
\text {... Senegal (from } 1 \text { to } 4 \text { ) }\end{array}$ & $\begin{array}{c}2.969 \\
(\mathrm{~N}=127)\end{array}$ & $\begin{array}{c}3.175 \\
(\mathrm{~N}=338)\end{array}$ & $\begin{array}{l}0.206^{* *} \\
(\mathrm{~N}=465)\end{array}$ & $\begin{array}{l}0.217^{* *} \\
(\mathrm{~N}=295)\end{array}$ \\
\hline (7) Burial: $=1$ if the respondent wants to be buried in Africa, $=0$ otherwise & $\begin{array}{c}0.437 \\
(\mathrm{~N}=103)\end{array}$ & $\begin{array}{c}0.767 \\
(\mathrm{~N}=287)\end{array}$ & $\begin{array}{l}0.330^{* * *} \\
(\mathrm{~N}=390)\end{array}$ & $\begin{array}{l}0.386^{* * *} \\
(\mathrm{~N}=250)\end{array}$ \\
\hline (8) Home: $=1$ if the respondent owns a home in Africa, $=0$ otherwise & $\begin{array}{c}0.795 \\
(\mathrm{~N}=127)\end{array}$ & $\begin{array}{c}0.891 \\
(\mathrm{~N}=339)\end{array}$ & $\begin{array}{l}0.096^{* *} \\
(\mathrm{~N}=466)\end{array}$ & $\begin{array}{l}0.134^{* *} \\
(\mathrm{~N}=295)\end{array}$ \\
\hline $\begin{array}{l}\text { (9) Sick mother=disapproval of a Senegalese student who does not renounce a scholarship... } \\
\text {... in a top French university to take care of a sick mother in Senegal (from } 1 \text { to } 3 \text { ) }\end{array}$ & $\begin{array}{c}2.276 \\
(\mathrm{~N}=127)\end{array}$ & $\begin{array}{c}2.361 \\
(\mathrm{~N}=332)\end{array}$ & $\begin{array}{c}0.085 \\
(\mathrm{~N}=459)\end{array}$ & $\begin{array}{c}0.096 \\
(\mathrm{~N}=292)\end{array}$ \\
\hline
\end{tabular}

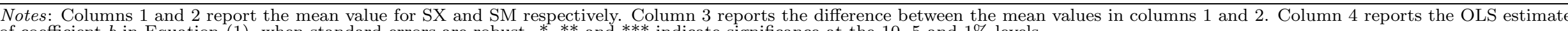
of coefficient $b$ in Equation (1), when standard errors are robust. ${ }^{*}, * *$ and $* * *$ indicate significance at the 10,5 and $1 \%$ levels. 
Table 2a: Comparing average SM/SX identification with the French culture and society.

\begin{tabular}{|c|c|c|c|c|}
\hline & $\begin{array}{l}\text { SX } \\
(\mathrm{a})\end{array}$ & $\begin{array}{l}\mathrm{SM} \\
(\mathrm{b})\end{array}$ & $\begin{array}{l}\text { Raw diff. } \\
\text { (b)-(a) }\end{array}$ & $\begin{array}{l}\text { Regression-adjusted diff. } \\
\text { (b)-(a) }\end{array}$ \\
\hline (1) Sympathy: sympathy of the respondent toward French people (from 1 to 10 ) & $\begin{array}{c}7.619 \\
(\mathrm{~N}=126)\end{array}$ & $\begin{array}{c}7.404 \\
(\mathrm{~N}=329)\end{array}$ & $\begin{array}{c}-0.215 \\
(\mathrm{~N}=455)\end{array}$ & $\begin{array}{l}-0.673^{* * *} \\
(\mathrm{~N}=291)\end{array}$ \\
\hline $\begin{array}{l}\text { (2) No conflict: }=1 \text { if the respondent believes immigrants should do whatever... } \\
\text {.. possible to avoid conflict with the hosting society, }=0 \text { otherwise }\end{array}$ & $\begin{array}{c}0.593 \\
(\mathrm{~N}=123)\end{array}$ & $(\mathrm{N}=326)$ & $\begin{array}{l}-0.093^{*} \\
(\mathrm{~N}=449)\end{array}$ & $\begin{array}{l}-0.135^{* *} \\
(\mathrm{~N}=286)\end{array}$ \\
\hline $\begin{array}{l}\text { (3) Best friend: }=1 \text { if the grandparents of the respondent's best friend originate from France, } \ldots \\
\ldots=0 \text { otherwise }\end{array}$ & $\begin{array}{l}0.394 \\
(\mathrm{~N}=127)\end{array}$ & $\begin{array}{c}0.201 \\
(\mathrm{~N}=328)\end{array}$ & $\begin{array}{l}-0.193^{*} * * \\
(\mathrm{~N}=455)\end{array}$ & $\begin{array}{c}-0.262^{* * *} \\
(\mathrm{~N}=290)\end{array}$ \\
\hline (4) Left-Right: $=1$ if the respondent's political preferences can be positioned on... & 0.752 & 0.615 & $-0.137 * * *$ & $-0.176^{* * *}$ \\
\hline ... a typical left-wing/right-wing scale, $=0$ otherwise & $(\mathrm{N}=117)$ & $(\mathrm{N}=312)$ & $(\mathrm{N}=429)$ & $(\mathrm{N}=276)$ \\
\hline $\begin{array}{l}\text { (5) Commonalities: commonalities the respondent believes she shares... } \\
\text {... with French people (from } 1 \text { to } 4 \text { ) }\end{array}$ & $\begin{array}{c}2.888 \\
(\mathrm{~N}=125)\end{array}$ & $\begin{array}{c}2.713 \\
(\mathrm{~N}=335)\end{array}$ & $\begin{array}{l}-0.175^{* *} \\
(\mathrm{~N}=460)\end{array}$ & $\begin{array}{l}-0.173^{*} \\
(\mathrm{~N}=292)\end{array}$ \\
\hline (6) Burial. -1 if the resnondent wants to be buriod in France -0 otherwice & 0.485 & 0.160 & $-0.325 * * *$ & $-0.357 * * *$ \\
\hline (7) & $(\mathrm{N}=103)$ & $(\mathrm{N}=287)$ & $(\mathrm{N}=390)$ & $(\mathrm{N}=250)$ \\
\hline $\begin{array}{l}\text { (7) Hide father: approval of a man of Senegalese origin who hides from his white... } \\
\text {... son's friends so that they may believe the son is a rooted French (from } 1 \text { to } 3 \text { ) }\end{array}$ & $\begin{array}{c}1.325 \\
(\mathrm{~N}=126)\end{array}$ & $\begin{array}{c}1.203 \\
(\mathrm{~N}=335)\end{array}$ & $\begin{array}{l}-0.122^{* * *} \\
(\mathrm{~N}=461)\end{array}$ & $\begin{array}{c}-0.223^{* * *} \\
(\mathrm{~N}=294)\end{array}$ \\
\hline
\end{tabular}

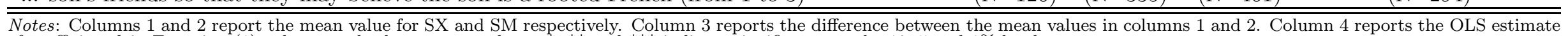
of coefficient $b$ in Equation (1), when standard errors are robust. ${ }^{*}, * *$ and $* * *$ indicate significance at the 10,5 and $1 \%$ levels.

Table 2b: Comparing average SM/SX secularization.

\begin{tabular}{|c|c|c|c|c|}
\hline & $\begin{array}{l}\text { SX } \\
(\mathrm{a})\end{array}$ & $\begin{array}{l}\mathrm{SM} \\
(\mathrm{b})\end{array}$ & $\begin{array}{l}\text { Raw diff. } \\
\text { (b)-(a) }\end{array}$ & $\begin{array}{l}\text { Regression-adjusted diff. } \\
\text { (b)-(a) }\end{array}$ \\
\hline & 7.592 & 9.006 & $1.414^{* * *}$ & $1.456^{* * *}$ \\
\hline (1) God: importance of god in the respondent's life (trom 1 to 10 ) & $(\mathrm{N}=125)$ & $(\mathrm{N}=330)$ & $(\mathrm{N}=455)$ & $(\mathrm{N}=291)$ \\
\hline (2) Exogamy: disapproval of one's child if he/she married a religious other (from 1 to 3 ) & 1.880 & 2.075 & $0.195^{* * *}$ & $0.174^{* *}$ \\
\hline & $\left(\begin{array}{c}\mathrm{N}=125) \\
8073\end{array}\right.$ & $\left(\begin{array}{c}\mathrm{N}=335) \\
8360\end{array}\right.$ & $(\mathrm{N}=460)$ & $(\mathrm{N}=293)$ \\
\hline (3) Sympathy: sympathy of the respondent toward people sharing the same religion (from 1 to 10) & $\begin{array}{c}8.073 \\
(\mathrm{~N}=124)\end{array}$ & $\begin{array}{c}8.360 \\
(\mathrm{~N}=331)\end{array}$ & $(\mathrm{N}=455)$ & $\begin{array}{c}0.331 \\
(\mathrm{~N}=291)\end{array}$ \\
\hline (4) Best friend: $=1$ if the respondent's best friend is of the same religion, $=0$ otherwise & $\begin{array}{c}0.589 \\
(\mathrm{~N}=124)\end{array}$ & $\begin{array}{c}0.675 \\
(\mathrm{~N}=320)\end{array}$ & $\begin{array}{l}0.086^{*} \\
(\mathrm{~N}=444)\end{array}$ & $\begin{array}{l}0.107^{*} \\
(\mathrm{~N}=287)\end{array}$ \\
\hline (5) Association: $=1$ if the association the respondent belongs to is related to religion, $=0$ otherwise & $\begin{array}{c}0.071 \\
(\mathrm{~N}=42)\end{array}$ & $\begin{array}{c}0.127 \\
(\mathrm{~N}=134)\end{array}$ & $\begin{array}{c}0.056 \\
\mathrm{~N}=176)\end{array}$ & $\begin{array}{c}0.048 \\
(\mathrm{~N}=127)\end{array}$ \\
\hline $\begin{array}{l}\text { (6) Commonalities: commonalities the respondent believes she shares... } \\
\text {... with people of the same religion (from } 1 \text { to } 4 \text { ) }\end{array}$ & $\begin{array}{c}2.912 \\
(\mathrm{~N}=125)\end{array}$ & $\begin{array}{c}3.190 \\
(\mathrm{~N}=336)\end{array}$ & $\begin{array}{l}0.278^{* * *} \\
(\mathrm{~N}=461)\end{array}$ & $\begin{array}{l}0.272^{* *} \\
(\mathrm{~N}=291)\end{array}$ \\
\hline
\end{tabular}

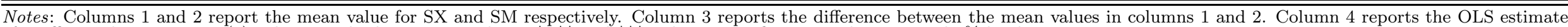
of coefficient $b$ in Equation (1), when standard errors are robust. ${ }^{*}, * *$ and $* * *$ indicate significance at the 10,5 and $1 \%$ levels. 
Table 3: Comparing the assimilation pattern over time of SM and SX. OLS analysis.

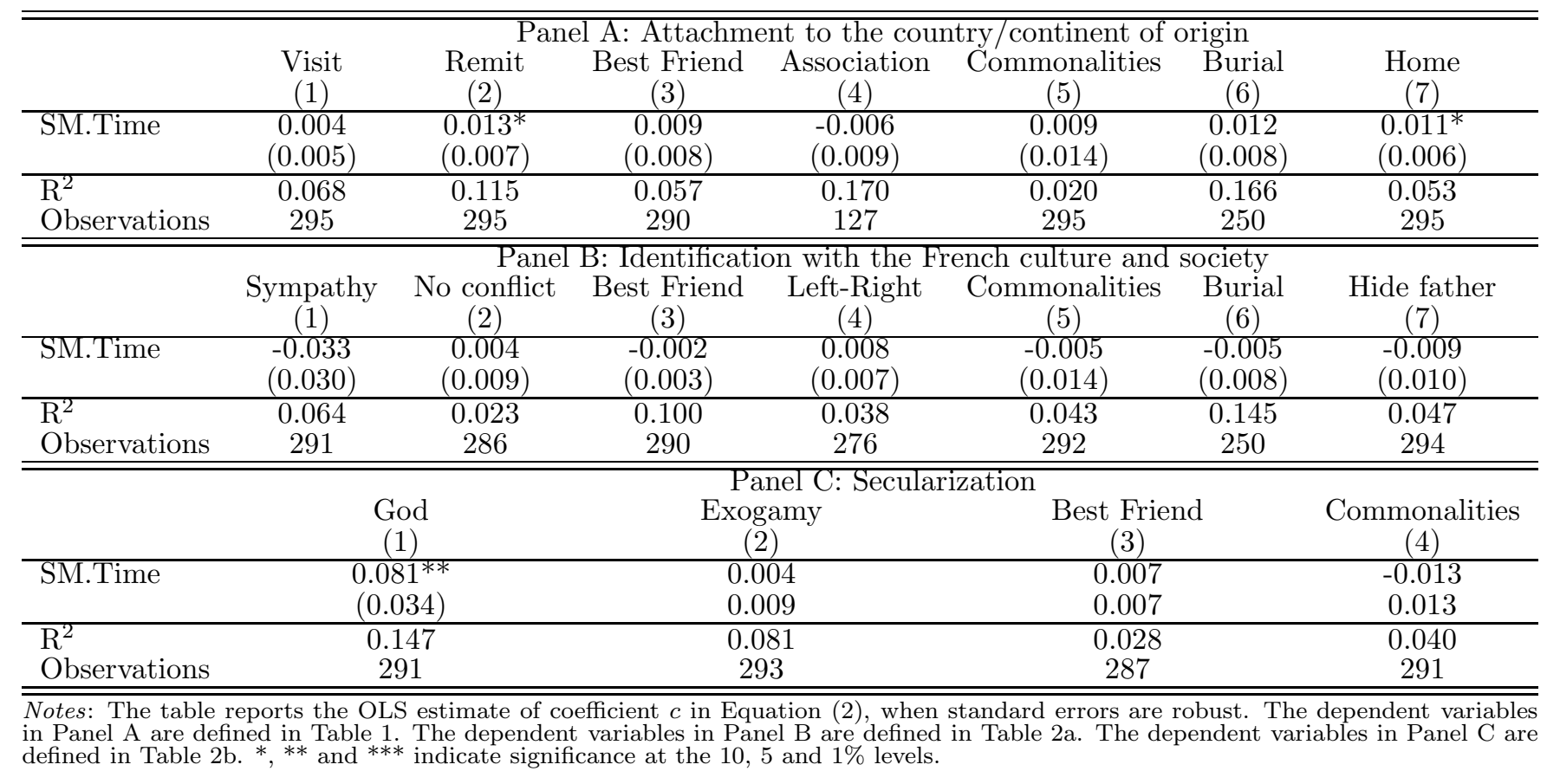


Table 4: Socioeconomic characteristics of SM and SX participants in our 2009 experiments. Difference of means analysis.

\begin{tabular}{l|c|c|c}
\hline Variable & $\begin{array}{c}\text { SM } \\
(\mathrm{a})\end{array}$ & $\begin{array}{c}\text { SX } \\
(\mathrm{b})\end{array}$ & $\begin{array}{c}\text { Difference } \\
(\mathrm{b}-\mathrm{a})\end{array}$ \\
\hline \multirow{2}{*}{ Female } & 0.50 & 0.55 & +0.05 \\
& $(\mathrm{~N}=16)$ & $(\mathrm{N}=11)$ & $\mathrm{p}=0.83$ \\
\hline \multirow{2}{*}{ Age } & 33.19 & 31.45 & -1.74 \\
& $(\mathrm{~N}=16)$ & $(\mathrm{N}=11)$ & $\mathrm{p}=0.59$ \\
\hline \multirow{2}{*}{ Education } & 7.33 & 7.63 & +0.30 \\
& $(\mathrm{~N}=15)$ & $(\mathrm{N}=8)$ & $\mathrm{p}=0.83$ \\
\hline \multirow{2}{*}{ Household income } & 3.79 & 4.00 & +0.21 \\
& $(\mathrm{~N}=14)$ & $(\mathrm{N}=9)$ & $\mathrm{p}=0.85$ \\
\hline \multirow{2}{*}{ Religiosity } & 2.60 & 4.90 & +2.30 \\
& $(\mathrm{~N}=15)$ & $(\mathrm{N}=10)$ & $\mathrm{p}=0.00$ \\
\hline \multirow{2}{*}{ Knows players from previous game sessions } & 0.43 & 0.36 & -0.07 \\
& $(\mathrm{~N}=16)$ & $(\mathrm{N}=11)$ & $\mathrm{p}=0.71$ \\
\hline
\end{tabular}

Notes: The table reports arithmetic means for the sub-samples of SM and SX players, and two-tailed t-tests assuming unequal variances. "Female" takes the value 1 if the participant is female and 0 otherwise. "Age" is equal to the age of the participant. "Education" ranges from 1 (less than primary school completed) to 10 (higher than college degree completed).

"Household income" ranges from 1 (less than 500 euros monthly) to 11 (more than 7,500 euros monthly). "Religiosity" ranges from 1 (never attends religious services) to 7 (attends religious services several times a week). "Knows players from previous sessions" takes the value 1 if the participant knows players who participated in previous game sessions and 0 otherwise. 
Table 5: Position of "FFF" respondents to the 2009 ESS and of FFF participants in our 2009 experiments on a left wing-right wing scale. Difference of means analysis.

\begin{tabular}{|c|c|c|}
\hline "FFF" respondents to the 2009 ESS & FFF participants in our 2009 experiment & $\begin{array}{c}\text { Diff } \\
(\mathrm{b}-\mathrm{a})\end{array}$ \\
\hline 1.94 & $(\mathrm{~b})$ & $-0.52^{* * *}$ \\
$(\mathrm{~N}=64)$ & $(\mathrm{N}=19)$ & $\mathrm{p}=0.01$ \\
\hline
\end{tabular}

Notes: The table reports a difference of means analysis. The variable of interest captures the position of respondents on a left wing-right wing scale. It ranges from 1 to 3 , where 1 means "more leftist than rightist", 2 means "in-between" and 3 means "more rightist than leftist." **, ** and *** indicate significance at the 10, 5 and $1 \%$ levels.

Table 6: Position of "FFF" respondents to the 2009 ESS and of FFF participants in our 2009 experiment on a left wing-right wing scale. OLS analysis.

\begin{tabular}{lccccc}
\hline \hline & \multicolumn{3}{c}{ Dep. var.: } & Position on a left wing-right wing scale \\
& $(1)$ & $(2)$ & $(3)$ & $(4)$ & $(5)$ \\
\hline (1) European Social Survey & $0.516^{* * *}$ & $0.522^{* * *}$ & $0.517^{* * *}$ & $0.473^{* *}$ & $0.447^{* *}$ \\
& $(0.177)$ & $(0.176)$ & $(0.179)$ & $(0.191)$ & $(0.198)$ \\
(2) Female & & 0.063 & 0.054 & 0.046 & -0.007 \\
& & $(0.183)$ & $(0.202)$ & $(0.203)$ & $(0.211)$ \\
(3) Age & & 0.001 & -0.001 & 0.000 \\
& & & $(0.006)$ & $(0.006)$ & $(0.007)$ \\
(4) Education & & & -0.093 & -0.050 \\
& & & & $(0.105)$ & $(0.111)$ \\
(5) Household income & & & & -0.130 \\
& & & & $0.089)$ \\
\hline $\mathrm{R}^{2}$ & 0.065 & 0.066 & 0.066 & 0.074 & 0.110 \\
Observations & 83 & 83 & 83 & 83 & 75 \\
\hline \hline
\end{tabular}

Notes: The table reports OLS estimates. The unit of observation is the respondent. The dependent variable ranges from 1 to 3 , where 1 means "more leftist than rightist", 2 means "in-between" and 3 means "more rightist than leftist." "European Social Survey" takes the value 1 if the individual is a respondent to the 2009 ESS, and 0 if she is a participant in our 2009 experiments. "Female" takes

the value 1 if the individual is female and 0 otherwise. "Age" is equal to the age of the individual.

"Education" ranges from 1 (less than lower secondary completed) to 4 (post secondary completed).

"Household income" ranges from 1 (first quintile) to 5 (fifth quintile). Standard errors are robust. *,

$* *$ and $* * *$ indicate significance at the 10,5 and $1 \%$ levels. 
Table 7: First names of the 2009 SM and SX players.

\begin{tabular}{|c|c|}
\hline First names of SM players & First names of SX players \\
\hline AMADOU & CECILE \\
AMIE & CHRISTINE \\
ASTOU & DANIEL \\
AWA & EPHIGENIE \\
CIRE & GASTON \\
FATOUMATA & HELENE \\
IBOU & LOUIS \\
IBRAHIMA & MAMDOU JEAN \\
KALS & NINA \\
KHADY & ROBERT ANTOINE \\
MAMADOU LAMINE & THERESE \\
MOUSTAPHA & \\
NDEYE & \\
OUSMANE & \\
SIDY & \\
SIRE & \\
TAMSIR & \\
YACINE & \\
\hline
\end{tabular}

Figure 1: Sample screenshot for the 2010 Names Game.

Comportement de Jeux des Habitants de l'Ile de France

\section{MOUSTAPHA}

Selon vous, quelle est la tradition religieuse de cette personne?

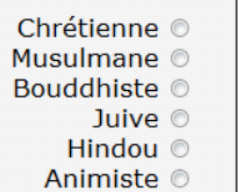

Selon vous, de quelle région cette personne est-elle originaire?

Amérique du Nord $\odot$ Amérique Latine et Caraïbes $\odot$ Afrique du Nord et Moyen Orient Afrique Noire

Europe 0 Asie $\bigcirc$

\section{Abandonner Continuer}

Translation of Figure 1: "What do you think is the religious heritage of this person (Christian, Muslim, Buddhist, Jewish, Hindu, Animist)? Which region do you think this person comes from (North America, Latin America and the Caribbean, North Africa and the Middle East, Sub-Saharan Africa, Europe, Asia)?"

Table 8: Categorization of 2009 SM players' first names as SMM or SMA by our 2010 FFF players.

\begin{tabular}{|c|c|}
\hline First names of SMM players & First names of SMA players \\
\hline AMADOU & AMIE \\
AWA & ASTOU \\
FATOUMATA & CIRE \\
IBRAHIMA & IBOU \\
KHADY & KALS \\
MAMADOU LAMINE & SIDY \\
MOUSTAPHA & SIRE \\
NDEYE & TAMSIR \\
OUSMANE & \\
YACINE & \\
\hline
\end{tabular}


Table 9: Amount sent by the sender in the 2009 simultaneous trust game. OLS analysis.

\begin{tabular}{|c|c|c|c|c|c|c|c|c|}
\hline & $(1)$ & $(2)$ & $\begin{array}{l}\text { Dep. } \\
(3)\end{array}$ & $\begin{array}{c}\text { ar.: Amount } \\
(4)\end{array}$ & $\begin{array}{c}\text { sent by the } \\
(5)\end{array}$ & $\begin{array}{r}\text { sender } \\
(6)\end{array}$ & $(7)$ & $(8)$ \\
\hline (1) $\mathrm{FFF} \rightarrow \mathrm{SM}$ & -0.071 & & -0.166 & & -0.352 & & -0.232 & \\
\hline (2) $\mathrm{FFF} \rightarrow \mathrm{SMM}$ & & -0.062 & & -0.220 & & -0.543 & & -0.326 \\
\hline (3) $\mathrm{FFF} \rightarrow \mathrm{SMA}$ & & -0.083 & & -0.098 & & -0.117 & & -0.108 \\
\hline (4) $\mathrm{SX} \rightarrow$ All & $-0.675^{* * *}$ & $-0.675 * * *$ & $-0.614^{* * *}$ & $-0.614^{* * *}$ & $-1.011^{* * *}$ & $-0.988^{* * *}$ & $-0.670^{* *}$ & $-0.656^{* *}$ \\
\hline (5) $\mathrm{SM} \rightarrow$ All & $-0.801^{* * *}$ & $-0.801^{* * *}$ & $-0.853^{* * *}$ & $-0.855^{* * *}$ & $-1.437^{* * *}$ & $-1.443^{* * *}$ & $-1.071^{* * *}$ & $-1.066^{* * *}$ \\
\hline \multicolumn{9}{|l|}{ SES of the sender } \\
\hline (6) Female & & & & & -0.516 & $-0.526^{*}$ & $-0.413^{*}$ & $-0.402^{*}$ \\
\hline (8) Household income & & & & & 0.029 & 0.033 & 0.033 & 0.035 \\
\hline (9) Education & & & & & -0.020 & -0.024 & -0.030 & -0.031 \\
\hline (10) Religiosity & & & & & -0.058 & -0.060 & -0.053 & -0.055 \\
\hline (11) Knows players from previous sessions & & & & & $0.824^{* * *}$ & $0.856^{* * *}$ & $0.632^{* * *}$ & $0.641^{* * *}$ \\
\hline \multicolumn{9}{|l|}{ SES of the receiver } \\
\hline (12) Female & & & & & -0.038 & -0.031 & -0.106 & -0.113 \\
\hline (13) Age & & & & & 0.005 & 0.003 & 0.004 & 0.003 \\
\hline (14) Household income & & & & & 0.026 & 0.028 & 0.018 & 0.020 \\
\hline (16) Religiosity & & & & & -0.034 & -0.034 & -0.030 & -0.029 \\
\hline (17) Knows players from previous sessions & & & & & 0.019 & 0.001 & -0.014 & -0.024 \\
\hline P-value of the test: $(1)=0$ & 0.60 & & 0.26 & & 0.11 & & 0.27 & \\
\hline P-value of the test: $(2)=0$ & & 0.77 & & 0.37 & & 0.11 & & 0.26 \\
\hline P-value of the test: $(3)=0$ & & 0.64 & & 0.61 & & 0.62 & & 0.66 \\
\hline P-value of the test: $(4)=(5)$ & 0.66 & 0.66 & 0.22 & 0.22 & 0.02 & 0.01 & 0.02 & 0.02 \\
\hline Session fixed effects & $\mathrm{No}$ & $\mathrm{No}$ & Yes & Yes & Yes & Yes & Yes & Yes \\
\hline Multiple Imputation Analysis & No & No & No & No & No & No & Yes & Yes \\
\hline $\mathrm{R}^{2}$ & 0.117 & 0.117 & 0.293 & 0.294 & 0.474 & 0.479 & 0.382 & 0.384 \\
\hline Observations & 178 & 178 & 178 & 178 & 134 & 134 & 178 & 178 \\
\hline
\end{tabular}

Notes: The table reports OLS estimates. The unit of observation is a dyad formed of FFF and SM or SX players. The dependent variable ranges from 0 (the sende sends nothing to the receiver to 3 (the sender sends her total endowment to the receiver). "FFF $\rightarrow$ SM" takes the value 1 if the sender is FFF and the receiver is SM, and 0 otherwise. "FFF $\rightarrow$ SMM" takes the value 1 if the sender is FFF and the receiver is SMM, and 0 otherwise. "FFF $\rightarrow$ SMA" takes the value 1 if the sender is FFF and the receiver is SMA, and 0 otherwise. "SM $\rightarrow$ All" takes the value 1 if the sender is SM and the receiver is any game partner, and 0 otherwise. "SX $\rightarrow$ All" takes the value 1 if the sender is SX and the receiver is any game partner, and 0 otherwise. The omitted category is the dummy "FFF $\rightarrow$ SX" that takes the value 1 if "Ele sender is FFF and the 1 (leivis is monthly) to 11 (more than 7,500 euros monthly). "Religiosity" ranges from 1 (never attends religious services) to 7 (attends religious services several times a week) "Knows players from previous sessions" takes the value 1 if the player knows players who participated in previous game sessions and 0 otherwise. Standard errors (not reported here due to space constraints) are clustered at the sender level. ${ }^{*},{ }^{* *}$ and ${ }^{* * *}$ indicate significance at the 10,5 and $1 \%$ levels. 
Table 10: Amount sent back by the receiver in the 2009 simultaneous trust game. OLS analysis.

\begin{tabular}{|c|c|c|c|c|c|c|c|c|}
\hline & $(1)$ & $(2)$ & $\begin{array}{l}\text { ep. var.: } \\
(3)\end{array}$ & $\begin{array}{c}\text { Amoun } \\
(4)\end{array}$ & $\begin{array}{c}\text { sent back } \\
(5)\end{array}$ & $\begin{array}{l}y \text { the rece } \\
(6)\end{array}$ & er $(7)$ & $(8)$ \\
\hline (1) $\mathrm{FFF} \rightarrow \mathrm{SM}$ & $-0.092^{*}$ & & -0.076 & & $-0.243^{* * *}$ & & $-0.149^{*}$ & \\
\hline (2) $\mathrm{FFF} \rightarrow \mathrm{SMM}$ & & $-0.152^{*}$ & & -0.161 & & $-0.331^{* *}$ & & $-0.251^{* *}$ \\
\hline (3) $\mathrm{FFF} \rightarrow \mathrm{SMA}$ & & -0.001 & & 0.052 & & -0.125 & & 0.022 \\
\hline (4) $\mathrm{SX} \rightarrow$ All & -0.031 & -0.031 & -0.023 & -0.021 & -0.076 & -0.070 & 0.032 & 0.050 \\
\hline (5) $\mathrm{SM} \rightarrow$ All & -0.117 & -0.117 & -0.097 & -0.099 & $-0.275^{* *}$ & $-0.282^{* *}$ & -0.128 & -0.121 \\
\hline \multicolumn{9}{|l|}{$\underline{\text { SES of the receiver }}$} \\
\hline (6) Female & & & & & 0.025 & 0.011 & 0.115 & 0.115 \\
\hline (7) Age & & & & & 0.001 & 0.002 & 0.002 & 0.003 \\
\hline (8) Household income & & & & & -0.010 & -0.009 & -0.004 & -0.002 \\
\hline (9) Education & & & & & 0.016 & 0.014 & -0.003 & -0.004 \\
\hline (10) Religiosity & & & & & -0.027 & -0.027 & -0.042 & $-0.044^{*}$ \\
\hline (11) Knows players from previous sessions & & & & & 0.069 & 0.081 & 0.107 & 0.113 \\
\hline \multicolumn{9}{|l|}{ SES of the sender } \\
\hline (12) Female & & & & & -0.093 & -0.096 & -0.039 & -0.049 \\
\hline (13) Age & & & & & 0.002 & 0.002 & 0.001 & 0.000 \\
\hline (14) Household income & & & & & 0.006 & 0.008 & 0.006 & 0.008 \\
\hline (15) Education & & & & & -0.022 & -0.024 & $-0.027^{*}$ & $-0.028^{*}$ \\
\hline (16) Religiosity & & & & & $-0.044^{* *}$ & $-0.044^{* *}$ & $-0.033^{*}$ & -0.031 \\
\hline (17) Knows players from previous sessions & & & & & $0.152^{*}$ & 0.135 & 0.084 & 0.061 \\
\hline P-value of the test: $(1)=0$ & 0.08 & & 0.24 & & 0.01 & & 0.07 & \\
\hline P-value of the test: $(2)=0$ & & 0.06 & & 0.11 & & 0.01 & & 0.02 \\
\hline P-value of the test: $(3)=0$ & & 0.99 & & 0.56 & & 0.22 & & 0.84 \\
\hline P-value of the test: $(4)=(5)$ & 0.37 & 0.37 & 0.36 & 0.34 & 0.09 & 0.07 & 0.10 & 0.08 \\
\hline Session fixed effects & $\overline{\mathrm{No}}$ & $\mathrm{No}$ & Yes & $\overline{\text { Yes }}$ & Yes & $\overline{\mathrm{Yes}}$ & Yes & Yes \\
\hline Multiple Imputation Analysis & No & No & No & No & No & No & Yes & Yes \\
\hline $\mathrm{R}^{2}$ & 0.021 & 0.030 & 0.128 & 0.143 & 0.244 & 0.257 & 0.194 & 0.216 \\
\hline Observations & 166 & 166 & 166 & 166 & 129 & 129 & 166 & 166 \\
\hline
\end{tabular}

Notes: The table reports OLS estimates. The unit of observation is a dyad formed of FFF and SM or SX players. The dependent variable ranges from 0 (the receiver sends back nothing to the sender) to 1 (the receiver sends back her total endowment to the sender). "FFF $\rightarrow$ SM" takes the value 1 if the receiver is FFF and the sender is SM, and 0 otherwise. "FFF $\rightarrow$ SMM" takes the value 1 if the receiver is FFF and the sender is SMM and 0 otherwise. "FFF $\rightarrow$ SMA" takes the value 1 if the receiver is FFF and the sender is SMA, and 0 otherwise. "SM $\rightarrow$ All" takes the value $1 \mathrm{i}$ the receiver is SM and the sender is any game partner, and 0 otherwise. "SX $\rightarrow$ FFF" takes the value 1 if the receiver is SX and the sender is any game partner, and "0 otherwise. The omitted category is the dummy "FFF $\rightarrow$ SX" that takes the value 1 if the receiver is FFF and the sender is SX, and 0 otherwise. "Female takes the value 1 if the player is female and otherwise. "Age" is equal to the age of the player. "Education" ranges ( 71 ( mo times a week). "Knows players from previous sessions" takes the value 1 if the player knows players who participated in previous game sessions and 0 otherwise. Standard errors (not reported here due to space constraints) are clustered at the receiver level. ${ }^{*}, * *$ and $* * *$ indicate significance at the 10,5 and $1 \%$ levels. 
Table 11: SM and SX guesses in the 2009 strategic dictator game. OLS analysis.

\begin{tabular}{|c|c|c|c|c|}
\hline & $\begin{array}{l}\text { Dep. var } \\
\text { (1) }\end{array}$ & $\begin{array}{l}\therefore \text { Guesses } \\
(2)\end{array}$ & $\begin{array}{l}\text { about dic } \\
(3)\end{array}$ & $\begin{array}{l}\text { s' donations } \\
\text { (4) }\end{array}$ \\
\hline (1) $\mathrm{SM} \rightsquigarrow \mathrm{FFF} \rightarrow \mathrm{SM}$ & $\begin{array}{l}-0.636^{*} \\
(0.368)\end{array}$ & $\begin{array}{c}-0.678^{* *} \\
(0.326)\end{array}$ & $\begin{array}{l}-1.062^{*} \\
(0.552)\end{array}$ & $\begin{array}{l}-0.729 \\
(0.497)\end{array}$ \\
\hline (2) $\mathrm{SM} \rightsquigarrow \mathrm{FFF} \rightarrow \mathrm{SX}$ & $\begin{array}{l}-0.303 \\
(0.377)\end{array}$ & $\begin{array}{l}-0.336 \\
(0.295)\end{array}$ & $\begin{array}{l}-0.765 \\
(0.516)\end{array}$ & $\begin{array}{l}-0.381 \\
(0.450)\end{array}$ \\
\hline (3) Female & & & $\begin{array}{c}0.129 \\
(0.820)\end{array}$ & $\begin{array}{c}0.954 \\
(0.655)\end{array}$ \\
\hline (4) Age & & & $\begin{array}{l}-0.006 \\
(0.024)\end{array}$ & $\begin{array}{l}-0.018 \\
(0.020)\end{array}$ \\
\hline (5) Household income & & & $\begin{array}{c}0.114^{* *} \\
(0.051)\end{array}$ & $\begin{array}{c}0.091 \\
(0.054)\end{array}$ \\
\hline (6) Education & & & $\begin{array}{c}-0.057 \\
(0.073)\end{array}$ & $\begin{array}{c}-0.079 \\
(0.074)\end{array}$ \\
\hline (7) Religiosity & & & $\begin{array}{c}0.036 \\
(0.126)\end{array}$ & $\begin{array}{l}-0.085 \\
(0.089)\end{array}$ \\
\hline (8) Knows players from previous sessions & & & $\begin{array}{c}-0.183 \\
(0.516)\end{array}$ & $\begin{array}{l}-0.095 \\
(0.313)\end{array}$ \\
\hline P-value of the test: $(1)=0$ & 0.09 & 0.05 & 0.07 & 0.16 \\
\hline P-value of the test: $(1)=(2)$ & 0.20 & 0.24 & 0.45 & 0.29 \\
\hline Session fixed effects & $\mathrm{No}$ & Yes & Yes & Yes \\
\hline Recipient face fixed effects & No & Yes & Yes & Yes \\
\hline Multiple Imputation Analysis & No & No & No & Yes \\
\hline $\mathrm{R}^{2}$ & 0.072 & 0.497 & 0.649 & 0.623 \\
\hline Observations & 41 & 41 & 34 & 41 \\
\hline
\end{tabular}

Notes: The table reports OLS estimates. The unit of observation is a triad formed of SM or SX guessers, FFF dictators and SM or SX recipients. The dependent variable ranges from 0 (the guesser guesses that the FFF dictator gives 0 euro to the recipient) to 5 (the guesser guesses that the FFF dictator gives 5 euros to the recipient). "SM $\rightsquigarrow \mathrm{FFF} \rightarrow \mathrm{SM}$ " takes the value 1 if the guesser is $\mathrm{SM}$ and the recipient is $\mathrm{SM}$, and 0 otherwise. "SM $\rightsquigarrow \mathrm{FFF} \rightarrow \mathrm{SX}$ " takes the value 1 if the guesser is SM and the recipient is SX, and 0 otherwise. The omitted category is the dummy "SX $\rightsquigarrow F F F \rightarrow S X$ " that takes the value 1 if the guesser is SX and the recipient is SX, and 0 otherwise. "Female" takes the value 1 if the guesser is female and 0 otherwise. "Age" is equal to the age of the guesser. "Education" ranges from 1 (less than primary school completed) to 10 (higher than college degree completed). "Household income" ranges from 1 (less than 500 euros monthly) to 11 (more than 7,500 euros monthly). "Religiosity" ranges from 1 (never attends religious services) to 7 (attends religious services several times a week). "Knows players from previous sessions" takes the value 1 if the guesser knows players who participated in previous game sessions and 0 otherwise. Standard errors are clustered at the guesser level. $*, * *$ and $* * *$ indicate significance at the 10,5 and $1 \%$ levels. 
Table 12: Comparing SM and SX distrust toward French institutions. OLS analysis.

\begin{tabular}{|c|c|c|c|c|c|c|c|}
\hline & $\begin{array}{c}\text { Schooling system } \\
\text { (1) }\end{array}$ & $\begin{array}{c}\text { Police } \\
(2)\end{array}$ & $\begin{array}{c}\text { Dep. var.: } \\
\text { Parliament } \\
(3)\end{array}$ & $\begin{array}{l}\text { Distrust toward F } \\
\text { Administration } \\
(4)\end{array}$ & $\begin{array}{l}\text { ench institutions } \\
\text { Judicial system } \\
(5)\end{array}$ & $\begin{array}{c}\text { Trade unions } \\
(6)\end{array}$ & $\begin{array}{l}\text { Private firms } \\
(7)\end{array}$ \\
\hline \multirow[t]{2}{*}{ (1) SM } & $0.380^{* * *}$ & 0.166 & $0.313^{* *}$ & $0.426^{* * *}$ & $0.357^{* * *}$ & $0.209^{* *}$ & $0.277^{* *}$ \\
\hline & $(0.088)$ & $(0.123)$ & $(0.123)$ & $(0.105)$ & $(0.119)$ & $(0.106)$ & $(0.108)$ \\
\hline \multirow[t]{2}{*}{ (2) Time } & $0.007^{*}$ & 0.004 & 0.008 & 0.003 & 0.005 & 0.007 & $0.011^{*}$ \\
\hline & $(0.004)$ & $(0.007)$ & $(0.007)$ & $(0.006)$ & $(0.006)$ & $(0.005)$ & $(0.006)$ \\
\hline \multirow[t]{2}{*}{ (3) Education } & -0.016 & -0.017 & -0.035 & -0.036 & $-0.066^{* *}$ & -0.008 & -0.025 \\
\hline & $(0.025)$ & $(0.033)$ & $(0.032)$ & $(0.029)$ & $(0.031)$ & $(0.030)$ & $(0.030)$ \\
\hline \multirow[t]{2}{*}{ (4) Female } & 0.101 & -0.136 & -0.087 & -0.003 & $-0.193^{*}$ & -0.173 & -0.027 \\
\hline & $(0.085)$ & $(0.114)$ & $(0.112)$ & $(0.103)$ & $(0.109)$ & $(0.105)$ & $(0.104)$ \\
\hline \multirow[t]{2}{*}{ (5) Age } & -0.004 & -0.009 & 0.005 & 0.003 & -0.002 & -0.005 & 0.014 \\
\hline & $(0.007)$ & $(0.010)$ & $(0.009)$ & $(0.009)$ & $(0.010)$ & $(0.009)$ & $(0.009)$ \\
\hline $\mathrm{R}^{2}$ & 0.073 & 0.018 & 0.043 & 0.060 & 0.067 & 0.031 & 0.062 \\
\hline Observations & 295 & 295 & 293 & 295 & 295 & 289 & 291 \\
\hline
\end{tabular}

Notes: The table reports OLS estimates. The unit of observation is the respondent. The dependent variables in columns $1,2,3,4,5,6$ and 7 capture distrust toward the schooling system, the police, the parliament, the administration, the judicial system, the trade unions and private firms respecrespondent is SM and 0 if the respondent is SX. The variable "Time" is continuous and captures the number of years elapsed since arrival of the first migrant in the respondent's family. "Education" captures the education of the first migrant. It ranges from 1 (no education) to 6 (higher education). "Female" takes the value 1 if the respondent is female and 0 if the respondent is male. "Age" is equal to the age of the respondent. Standard errors are robust. *, ** and $* * *$ indicate significance at the 10,5 and $1 \%$ levels. 
Table 13: Comparing SM and SX agreement with "French institutions treat individuals on an equal basis". OLS analysis.

\begin{tabular}{|c|c|c|c|c|c|c|}
\hline & \multicolumn{6}{|c|}{\begin{tabular}{l|l} 
Dep. var.: Agreement with "French institutions treat individuals on an equal basis"
\end{tabular}} \\
\hline & $\begin{array}{l}\text { Police } \\
\text { (1) }\end{array}$ & $\begin{array}{l}\text { Immigration authorities } \\
(2)\end{array}$ & $\begin{array}{c}\text { Prefecture } \\
(3)\end{array}$ & $\begin{array}{c}\text { Judicial system } \\
\text { (4) }\end{array}$ & $\underset{(5)}{\text { Schooling system }}$ & Pôle $\underset{(6)}{\text { Emploi }}$ \\
\hline \multirow[t]{2}{*}{ (1) SM } & $-0.218^{*}$ & $-0.265^{* *}$ & $-0.445^{* * *}$ & $-0.318^{* * *}$ & $-0.432^{* * *}$ & $-0.263^{* *}$ \\
\hline & $(0.125)$ & $(0.131)$ & $(0.135)$ & $(0.120)$ & $(0.111)$ & $(0.125)$ \\
\hline \multirow[t]{2}{*}{ (2) Time } & -0.005 & 0.001 & 0.005 & -0.008 & -0.007 & 0.004 \\
\hline & $(0.006)$ & $(0.007)$ & $(0.007)$ & $(0.007)$ & $(0.006)$ & $(0.007)$ \\
\hline \multirow[t]{2}{*}{ (3) Education } & 0.039 & 0.017 & 0.024 & 0.045 & $0.063^{*}$ & 0.018 \\
\hline & $(0.032)$ & $(0.033)$ & $(0.037)$ & $(0.033)$ & $(0.033)$ & $(0.033)$ \\
\hline \multirow[t]{2}{*}{ (4) Female } & 0.025 & $-0.222^{*}$ & -0.152 & 0.001 & -0.086 & -0.083 \\
\hline & $(0.107)$ & $(0.115)$ & $(0.123)$ & $(0.117)$ & $(0.112)$ & $(0.120)$ \\
\hline \multirow[t]{2}{*}{ (5) Age } & 0.011 & 0.007 & -0.013 & 0.009 & 0.004 & -0.014 \\
\hline & $(0.009)$ & $(0.010)$ & $(0.010)$ & $(0.010)$ & $(0.010)$ & $(0.010)$ \\
\hline $\mathrm{R}^{2}$ & 0.027 & 0.033 & 0.047 & 0.041 & 0.070 & 0.023 \\
\hline Observations & 293 & 286 & 289 & 293 & 292 & 274 \\
\hline
\end{tabular}

Notes: The table reports OLS estimates. The unit of observation is the respondent. The dependent variables capture whether the respondent considers that the following French institutions treat individuals on an equal basis: the police (column 1), the immigration authoritie (column 2), the prefecture (column 3), the judicial system (column 4), the schooling system (column 5), and the Pôle Emploi (column 6). Each dependent variable ranges from 1 to 4 where 1 stands for "fully disagrees" and 4 stands for "fully agrees". "SM" takes the value 1 if the the first mis SM and 0 ir the respondent is SX. The "Er" " (higher education). "Female" takes the value 1 if the respondent is female and 0 if the respondent is male. "Age" is equal to the age of the respondent. Standard errors are robust. $* * *$ and $* * *$ indicate significance at the 10,5 and $1 \%$ levels. 
Table 14: Comparing the assimilation in France of SM and SX first migrants. OLS analysis.

\begin{tabular}{|c|c|c|c|c|c|c|}
\hline & \multicolumn{6}{|c|}{\begin{tabular}{l|l} 
Dep. var.: Assimilation of SM and SX first migrants \\
\end{tabular}} \\
\hline & \multicolumn{2}{|c|}{ 1st mig. married a non-French } & \multicolumn{2}{|c|}{ 1st mig. married a co-rel. } & \multicolumn{2}{|c|}{ All ancestors married an African } \\
\hline & $(1)$ & $(2)$ & $(3)$ & $(4)$ & $(5)$ & $(6)$ \\
\hline (1) SM & $0.108^{* *}$ & -0.106 & $0.098^{*}$ & -0.029 & $0.117^{* *}$ & $0.418^{*}$ \\
\hline & $(0.045)$ & $(0.256)$ & $(0.052)$ & $(0.307)$ & $(0.050)$ & $(0.250)$ \\
\hline (2) SM.Time & & 0.000 & & -0.002 & & -0.005 \\
\hline & & $(0.006)$ & & $(0.007)$ & & $(0.006)$ \\
\hline (3) Time & 0.000 & 0.000 & -0.002 & -0.000 & -0.003 & 0.001 \\
\hline & $(0.002)$ & $(0.006)$ & $(0.002)$ & $(0.007)$ & $(0.003)$ & $(0.005)$ \\
\hline (4) SM.Age & & 0.007 & & 0.007 & & -0.004 \\
\hline & & $(0.008)$ & & $(0.009)$ & & $(0.009)$ \\
\hline (5) Education & $-0.031^{* * *}$ & $-0.031^{* * *}$ & -0.019 & -0.019 & -0.006 & -0.005 \\
\hline & $(0.010)$ & $(0.010)$ & $(0.013)$ & $(0.013)$ & $(0.012)$ & $(0.012)$ \\
\hline (6) Female & 0.031 & 0.031 & 0.067 & 0.065 & -0.004 & -0.005 \\
\hline & $(0.032)$ & $(0.033)$ & $(0.045)$ & $(0.045)$ & $(0.040)$ & $(0.039)$ \\
\hline (7) Age & $-0.007^{* *}$ & $-0.012^{*}$ & -0.004 & -0.009 & -0.000 & 0.002 \\
\hline & $(0.003)$ & $(0.007)$ & $(0.004)$ & $(0.008)$ & $(0.004)$ & $(0.008)$ \\
\hline$\overline{\mathrm{R}^{2}}$ & 0.109 & 0.116 & 0.046 & 0.048 & 0.037 & 0.043 \\
\hline Observations & 246 & 246 & 279 & 279 & 286 & 286 \\
\hline
\end{tabular}

Notes: The table reports OLS estimates. The unit of observation is the respondent. The dependent variable in columns 1 and 2 is a dummy that captures whether the first male migrants married a non-French woman. The dependent variable in columns 3 and 4 is a dummy that captures whether the first migrant married someone of the same religion. The dependent variable in columns 5 and 6 is a dummy that captures whether all the parents and grandparents of the respondent married someone of African background. "SM" takes the value 1 if the respondent is SM and 0 if the respondent is SX. The variable "Time" is continuous and captures the number of years elapsed since arrival of the first migrant in the respondent's family. "Education" captures the education of the first migrant. It ranges from 1 (no education) to 6 (higher education). "Female" takes the value 1 if the respondent is female and 0 if the respondent is male. "Age" is equal to the age of the respondent. Standard errors are robust. ${ }^{*},{ }^{* *}$ and $* * *$ indicate significance at the 10,5 and $1 \%$ levels.

Figure 2: Sample screenshot of the 2010 Beauty game.

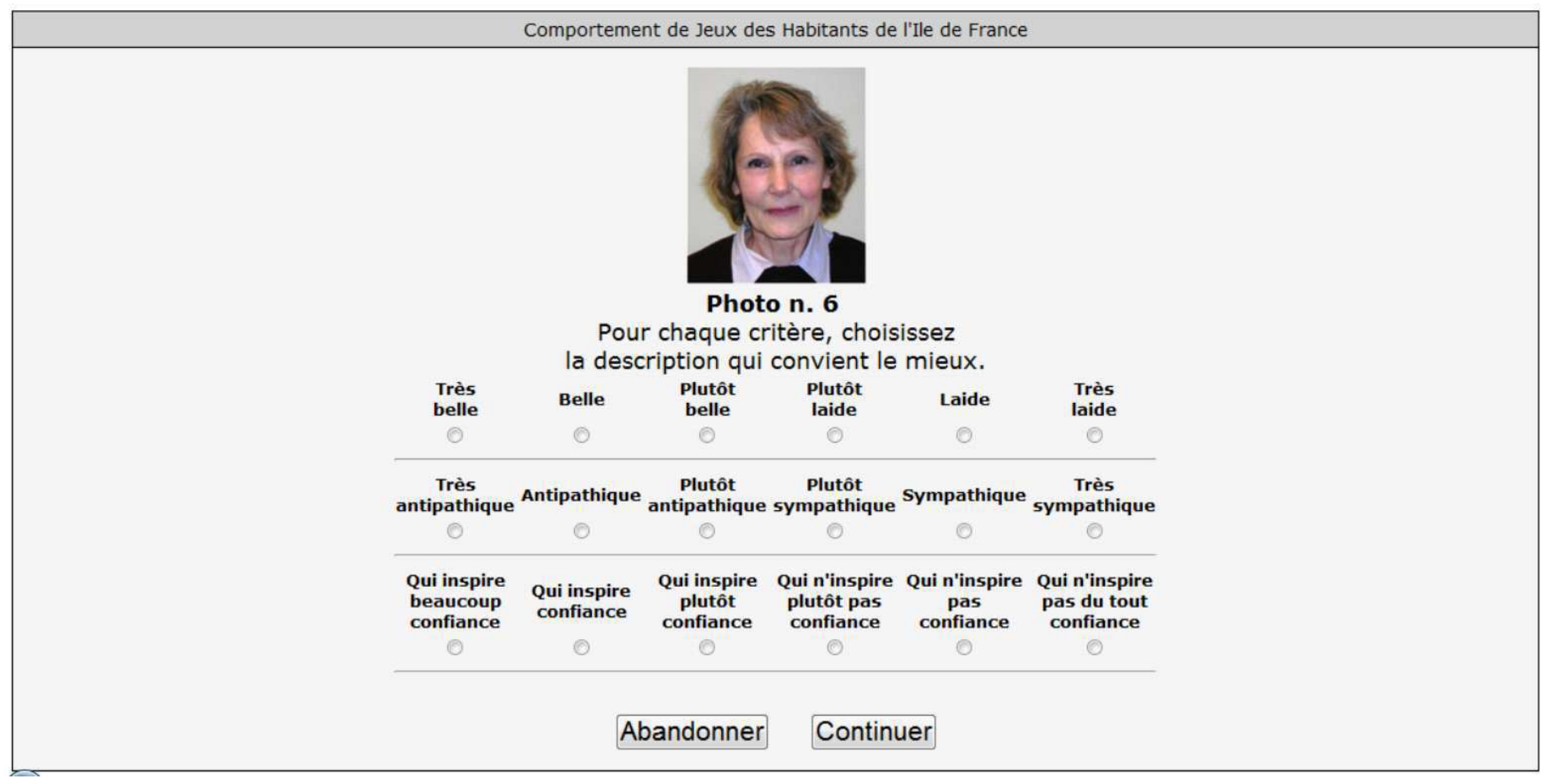

Translation of Figure 2: "For each pair of adjectives, choose the option that best corresponds to the photographed person: pretty/ugly ("very pretty", "pretty", "somewhat pretty", "somewhat ugly", "ugly", "very ugly"); friendly/unfriendly ("very friendly", "friendly", "somewhat friendly", "somewhat unfriendly", "unfriendly", "very unfriendly"); trustworthy/untrustworthy ("very trustworthy", "trustworthy", "somewhat trustworthy", "somewhat untrustworthy", "untrustworthy", "very untrustworthy")." 
Table 15: Amount sent back by the receiver in the 2009 simultaneous trust game, controlling for the beauty, friendliness and trustworthiness of the 2009 SM, SX and FFF players.

\begin{tabular}{|c|c|c|}
\hline & $\begin{array}{l}\text { Dep. var.: } \\
\text { (1) }\end{array}$ & $\begin{array}{l}\text { Amount sent back by the receiver } \\
(2)\end{array}$ \\
\hline (1) $\mathrm{FFF} \rightarrow \mathrm{SM}$ & $\begin{array}{c}-0.207^{* *} \\
(0.097)\end{array}$ & \\
\hline (2) $\mathrm{FFF} \rightarrow \mathrm{SMM}$ & & $\begin{array}{c}-0.298^{* *} \\
(0.118)\end{array}$ \\
\hline (3) $\mathrm{FFF} \rightarrow \mathrm{SMA}$ & & $\begin{array}{l}-0.031 \\
(0.126)\end{array}$ \\
\hline (4) $\mathrm{SX} \rightarrow$ All & $\begin{array}{c}0.103 \\
(0.131)\end{array}$ & $\begin{array}{c}0.136 \\
(0.129)\end{array}$ \\
\hline (5) $\mathrm{SM} \rightarrow$ All & $\begin{array}{l}-0.136 \\
(0.099)\end{array}$ & $\begin{array}{l}-0.120 \\
(0.101)\end{array}$ \\
\hline$\underline{\text { Facial traits of the receiver }}$ & & \\
\hline (6) Beauty & $\begin{array}{l}-0.009 \\
(0.097)\end{array}$ & $\begin{array}{l}-0.014 \\
(0.099)\end{array}$ \\
\hline (7) Friendliness & $\begin{array}{c}0.088 \\
(0.261)\end{array}$ & $\begin{array}{c}0.086 \\
(0.250)\end{array}$ \\
\hline (8) Trustworthiness & $\begin{array}{c}0.037 \\
(0.231)\end{array}$ & $\begin{array}{c}0.048 \\
(0.224)\end{array}$ \\
\hline$\underline{\text { Facial traits of the sender }}$ & & \\
\hline (9) Beauty & $\begin{array}{c}0.072 \\
(0.080)\end{array}$ & $\begin{array}{c}0.040 \\
(0.084)\end{array}$ \\
\hline (10) Friendliness & $\begin{array}{l}-0.155 \\
(0.241)\end{array}$ & $\begin{array}{l}-0.170 \\
(0.247)\end{array}$ \\
\hline (11) Trustworthiness & $\begin{array}{c}0.091 \\
(0.237) \\
\end{array}$ & $\begin{array}{c}0.131 \\
(0.246) \\
\end{array}$ \\
\hline P-value of the test: $(1)=0$ & 0.04 & \\
\hline P-value of the test: $(2)=0$ & & 0.02 \\
\hline P-value of the test: $(3)=0$ & & 0.81 \\
\hline P-value of the test: $(4)=(5)$ & 0.04 & 0.02 \\
\hline Sender/Receiver SES controls & Yes & Yes \\
\hline Session fixed effects & Yes & Yes \\
\hline Multiple Imputation Analysis & Yes & Yes \\
\hline $\mathrm{R}^{2}$ & 0.258 & 0.280 \\
\hline Observations & 141 & 141 \\
\hline
\end{tabular}

Notes: The table reports OLS estimates. The unit of observation is a dyad formed of FFF and SM or SX players. The dependent variable ranges from 0 (the receiver sends back nothing to the sender) to 1 (the receiver sends back her total endowment to the sender). "FFF $\rightarrow$ SM" takes the value 1 if the receiver is FFF and the sender is SM, and 0 otherwise. "FFF $\rightarrow$ SMM" takes the value 1 if the receiver is FFF and the sender is SMM, and 0 otherwise. "FFF $\rightarrow$ SMA" takes the value 1 if the receiver is FFF and the sender is SMA, and 0 otherwise. "SM $\rightarrow$ All" takes the value 1 if the receiver is SM and the sender is SM, SX or FFF, and 0 otherwise. "SX $\rightarrow$ FFF" takes the value 1 if the receiver is SX and the sender is SM, SX or FFF, and 0 otherwise. The omitted category is the dummy "FFF $\rightarrow$ SX" that takes the value 1 if the receiver is FFF and the sender is SX, and 0 otherwise. "Beauty" ranges from 1 to 6 where 1 stands for "very ugly" and 6 stands for "very beautiful". "Friendliness" ranges from 1 to 6 where 1 stands for "very unfriendly" and 6 stands for "very friendly". "Trustworthiness" ranges from 1 to 6 where 1 stands for "very untrustworthy" and 6 stands for "very trustworthy". Controls for the SES of the sender and of the receiver include their gender, age, household income, education, religiosity and whether they know players from previous sessions. Standard errors are clustered at the sender level. ${ }^{*}, * *$ and $* * *$ indicate significance at the 10,5 and $1 \%$ levels. 


\section{Appendix}

\section{Appendix A1: Evidence of SM/SX similarity before migration to France}

Ethnographic evidence lends support to the fact that families from the Joola and Serer communities who converted to Christianity in the 19th century were not different from those who converted to Islam during the same period: those who became Christians rather than Muslims were not characterized by propensities more consistent with the social mobility of their descendants (and therefore, among those descendants who would migrate to France after WWII, the ability to assimilate)

The historical record shows that the southwestern part of Senegal that is populated by the Joola and Serer communities was penetrated by both Muslim jihadists and Christian missionaries in the mid-19th century. Yet, these Joola and Serer communities resisted conversion from both world religions until the $1950 \mathrm{~s}$, when the economic returns to conversion became more attractive (Asante (2009) and Cannot-Brown (2009)). At that time, conversion to Islam or Christianity was indeed associated with religious networks allowing individuals to market their crops and access credit (see Gastellu (1981)). However, the economic returns to converting to one or the other religion were equal. Only the crops targeted by each religion were different: Muslim jihadists targeted crops they considered as "pure", thereby leaving the "impure" (but just as economically rewarding) ones to Christian missionaries. More specifically, while Muslim jihadists encouraged the development of the groundnut trade, Christian missionaries helped in the development of commerce in palm wine, an enterprise that was scorned by the Muslims (see Linares (1992)). In fact, Islam and Christianity implicitly shared the market for the two crops that, in this southwestern part of Senegal, were easiest to cultivate.

The ethnographic evidence suggests that the different integration patterns between SM and SX migrants in France in the 21 st century cannot be accounted for by the fact that their ancestors who converted to Islam and Christianity in the 19th century were different in terms of propensities toward economic success. Yet, it could be that differences across SM and SX emerged after conversion, notably with respect to their access to education. Catholic primary schools, because they benefited from greater support from the French colonial authorities, maybe constituted better stepping stones to higher education than did madrasa.

The survey that we conducted in 2009 among 511 second- and third-generation SM and SX immigrants to France (see subsection 2.1. for a description) rules out this possibility (at least for these SM and SX families who migrated to France). Our survey indeed shows that the first migrants in these SM and SX families are similar in terms of level of education and occupation in Senegal (i.e., before they migrated to France). As shown in rows 1 and 2 of Table A1, the difference in the highest level of education completed in Senegal and in the probability to be involved in on-farm rather than off-farm activities is not statistically significant across SM and SX first migrants. Notably, SM first migrants are not less educated nor more likely to be involved in on-farm activities in Senegal than are SX first migrants. Moreover, row 3 of Table A1 confirms that SM and SX first migrants arrived at the same time to France (i.e., in the 1970s). At that time, droughts in Senegal caused an agrarian crisis, and with relatively open borders to the former metropole considerable numbers of Serers and Joolas migrated, mostly to take jobs in the labor-hungry industrial suburbs of France's major cities.

Table A1: Education, occupation, and number of years since arrival to France of the first migrants in SM and SX families. Difference of means analysis.

\begin{tabular}{|c|c|c|c|}
\hline & $\begin{array}{l}\text { SX } \\
\text { (a) }\end{array}$ & $\begin{array}{l}\text { SM } \\
\text { (b) }\end{array}$ & $\begin{array}{l}\text { Difference } \\
\text { (b)-(a) }\end{array}$ \\
\hline (1) Highest level of education completed in Senegal (from 1 to 6) & $\begin{array}{c}3.160 \\
(\mathrm{~N}-106)\end{array}$ & 2.861 & -0.300 \\
\hline $\begin{array}{l}\text { (2) Occupation in Senegal: }=1 \text { if involved in on-farm activities, }=0 \text { if... } \\
\ldots \text { involved in off-farm activities }\end{array}$ & $\begin{array}{l}0.667 \\
(\mathrm{~N}=18)\end{array}$ & $\begin{array}{l}0.535 \\
(\mathrm{~N}=71)\end{array}$ & $\begin{array}{c}0.132 \\
(\mathrm{~N}=89)\end{array}$ \\
\hline (3) Number of years since arrival to France & $\begin{array}{c}39.053 \\
(\mathrm{~N}=94)\end{array}$ & $\begin{array}{c}39.429 \\
(\mathrm{~N}=233)\end{array}$ & $\begin{array}{c}0.376 \\
(\mathrm{~N}=327)\end{array}$ \\
\hline
\end{tabular}

Notes: Columns 1 and 2 report the mean value for SX and SM respectively. Column 3 reports the difference between the mean values in columns 1 and 2. The highest level of education completed in Senegal (row 1) is coded as follows: 1 if "no schooling", 2 if "primary or some primary", 3 if "vocational degree", 4 if "some secondary", 5 if "completed secondary" and 6 if "post-secondary". $*, * *$ and $* * *$ indicate significance at the 10,5 and $1 \%$ levels.

\section{Appendix A2: Description of the 2009 speed chatting game, voting game, dictator game and strategic dictator game}

\section{The 2009 speed chatting game}

The 2009 speed chatting game followed the simultaneous trust game. For this game, our ten players were placed into two teams of 5 , each following the same protocol. Each player on a team was instructed that he/she would have a few minutes to meet (and we emphasized, to get to know) each member of the other team, thereby "speed chatting" with five other players, sequentially, as in a speed-dating situation. After meeting each partner, players were given 1 minute to jot down notes on a piece of paper. After meeting all members of the other group, each player received a sheet of paper with the picture of each person he or she had just met, and a series of eight personal questions about them (their age, their religion, their job, whether they had obtained their Baccalauréat (the French high-school diploma), the country in which 
they were born, the district in which they live, whether they are married and their favorite hobby). Players were allowed to consult their notes. For each question, subjects provided their answer, or selected "don't know", and indicated whether they learned this information from their chat, or simply guessed the answer. For each correct answer, subjects earned 1 euro 1 Finally, players were asked to report any additional information they had learned about their interlocutor, as well as whether they believed they could be friends with this interlocutor and recommend this interlocutor to an employer. Figure A2-a illustrates a sample sheet for the speed chatting game.

Figure A2-a: Sample sheet of the 2009 speed chatting game.

\begin{tabular}{|c|c|c|}
\hline \multicolumn{3}{|c|}{$\begin{array}{l}\text { QUESTONNALE SUR LB JEU DE CONVERSA NON } \\
\text { PROJET \& COMPORTEMENTS DE JEU DES HABITANTS DE L'IL.E DE FRANCE } x \\
\text { (Sciences-Po Paric, Université Paris } 1 \text { Panthéon Sorbonne, Université de Stanford) }\end{array}$} \\
\hline \multicolumn{3}{|l|}{ Numéro didentification : } \\
\hline Questions & $\begin{array}{l}\text { Lavez-vous deviné } \\
\text { ou appris durant le } \\
\text { jeu de comversatoo? }\end{array}$ & 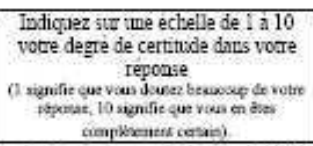 \\
\hline 1. Quel age a cette persoune? & Deviné / Appris & 12345678910 \\
\hline 2. Quelle est sa religion? & Devizé / Appris & 12345678910 \\
\hline $\begin{array}{l}\text { 3. Travaille-t-elle a son compte? } \\
\text { OUI NON }\end{array}$ & Detiné / Appris & 12345678910 \\
\hline $\begin{array}{l}\text { 4. A-t-elle le Baccalaurear? } \\
\text { OUI N NON }\end{array}$ & Deviné Appris & 12345678910 \\
\hline 5. Dans quel pays est-lle vée? & Desiué Appris & 12345678910 \\
\hline $\begin{array}{l}\text { 6. Daus quel anoudissement babite-t- } \\
\text { elle? Si elle n'habite pas Pans, marquez } \\
\text { Wp? }\end{array}$ & Deviné / Appris & 12345678910 \\
\hline 7 Est-elle maries? OUI / NON & Derizé / Appris & 123456789910 \\
\hline 8. Quel est son loisir preféré? & Deviné / Apprís. & 12345678910 \\
\hline
\end{tabular}

Translation of Figure A2-a: "1. How old is this person? 2. What is her religion? 3. Is she self-employed? 4. Does she hold a A-level? 5. What is her country of birth? 6. In which Parisian neighborhood does she live. Indicate "NP" if she does not live in Paris. 7. Is she married? 8. What is her hobby? [For each of these 10 questions, our players had to answer the two following questions:] Did you guess or learn this information? On a 1 to 10 scale, indicate how confident you are in your answer (1 means that you are not confident at all, 10 that you are fully confident)."

\section{The 2009 voting game}

The 2009 voting game took place after the speed chatting game. Each player in each of the two teams of five players formed during the speed chatting game was asked to play two roles, sequentially. First, each player was to be a voter, i.e. to choose one leader from the other team, knowing that the leader would have the responsibility of dividing 30 euros between herself and her electorate in any way she wanted (including keeping it all to herself). Each voter received a hand-out with the pictures of each of the five candidates she was to rank in order of preference for the role of leader. Second, each player was to imagine herself the leader: on a separate hand-out, each player had to indicate how much she would allocate to each of the members of her electorate, were she to be elected the leader. The player with the highest ranking in votes in each team became the leader, and her allocations were distributed between herself and the members of the other team.

The voting game was meant to measure taste-based discrimination and/or statistical discrimination after the speedchatting socialization phase. Clearly, the decision of the voter to rank member $i$ of the other team first can be motivated by unconditional altruism toward $i$ (i.e.: the willingness to increase the chance for $i$ of being elected and of getting 30 euros no matter how $i$ might allocate the award). It can also be motivated by trust, which is the belief that $i$ is most likely to return a larger share of her 30 euros to her electorate, or to certain members of her electorate. Similarly, the decision of the leader to allocate a strictly positive amount to member $i$ of the other team can also be motivated by unconditional altruism toward $i$ (i.e.: the willingness to increase $i$ 's payoff irrespective of $i$ 's electoral choice). It can also be motivated by belief-based reciprocal altruism, that is the willingness to reward $i$ based on the belief that $i$ voted for the leader.

\section{The 2009 dictator game}

After the voting game, subjects were ushered into a single room to play a dictator game All players were shown the same set of six partners (whom we call recipients) on a large screen revealing only their faces and ascribed first names,

\footnotetext{
${ }^{1}$ Subjects were told that the 1-euro award was independent of whether they learned the information from their chat or guessed it.

${ }^{2}$ In this classic experimental game introduced by Kahneman, Knetsch and Thaler (1986), players view pictures of people whom they have

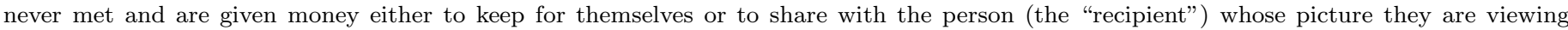


which we strategically altered as is commonly done in correspondence tests conducted by economists (see Bertrand and Mullainathan (2004)). More precisely, among the six recipients, two were apparent FFF with Christian names, two were ambiguous with alternatively Muslim and Christian names, such that players could reasonably think they were FFF with Christian names or North Africans with Muslim names, and two were apparent black Africans. For half of the sessions, subjects viewed one of the ambiguous recipients and one of the Senegalese recipients with a Christian name, and the other ambiguous recipient as well as the other Senegalese recipient with a Muslim name; for the other half of the sessions, this was reversed. By doing so, we avoid any confound between the ethnic type of the recipient and the face of the recipient. Put differently, the fact that donors see the same Senegalese face with alternated religious identities (one Christian, the other Muslim) allows us to run a within-face analysis. Figure A2-b illustrates the faces and alternating names of our recipients in the dictator game.

It is important to note that the four non-Senegalese recipients were recruited from the 19th district of Paris in a similar way as the 2009 players (while the Senegalese recipients, in order to assure ourselves that they would not be recognized by our Senegalese donors, were not residents of Ile de France). None of the recipients ever participated in our game sessions, and none was ever known personally by any of the donors.

The donors saw the sequence of recipients only once and were asked to make a decision to allocate $a=\{0,1,2,3,4,5\}$ euros to each recipient - out of 5 euros allotted to them each time, being assured that the amounts accruing to each recipient would actually be transferred to them. Given that the recipient has no say in the dictator game, differences in the dictator's donation to the various recipients is commonly interpreted as differences in unconditional altruism.

At this stage, it is critical to recall the professional norm among experimental economists that proscribes the use of deception in the lab. Deception indeed compromises the ability of researchers to find participants who trust their experimental design (see Hertwig and Ortmann (2001) and Jamison, Karlan and Schechter (2008) for evidence that deception does affect participants' future behavior). Institutional review boards (IRBs) are the main enforcers of the "no deception" rule. More precisely, these boards are in charge of attesting that all procedures described in a protocol will be carried out exactly as stated in the instructions to the subjects and that all allocations of money that will be made in the experiment will be paid in exactly the amount chosen by the subjects. Our experimental protocol (and notably the section related to the 2009 dictator game) complies with this requirement and was therefore endorsed by the Stanford IRB. In the 2009 dictator game, we indeed told our subjects that the recipients whose pictures were shown were real people, recruited the same way as our subjects, which was true. We also assured our subjects that we would transfer to the recipients exactly the amounts chosen by our subjects, which we did. Finally, we did not say a word about the names of our recipients. We never claimed that the nature of the recipients' names was real and therefore did not deceive our subjects 3

Figure A2-b: Variations in the ethno-religious identity of the recipients in the 2009 dictator game.

\begin{tabular}{|c|c|c|c|c|c|c|c|}
\hline \multirow{2}{*}{ First name } & Version 1 & Sylvie & Georges & Khadija & Jean-Marc & Farida & Michel \\
\cline { 2 - 8 } & Version 2 & Sylvie & Mohammed & Joséphine & Jean-Marc & Christine & Aboubacar \\
\hline \multirow{2}{*}{$\begin{array}{c}\text { Ethnicity/ } \\
\text { Religion }\end{array}$} & Version 1 & FFF & FFF & SM & FFF & $\begin{array}{c}\text { Muslim North } \\
\text { African }\end{array}$ & SX \\
\cline { 2 - 9 } & Version 2 & FFF & $\begin{array}{c}\text { Muslim North } \\
\text { African }\end{array}$ & SX & FFF & FFF & SM \\
\hline
\end{tabular}

\section{The 2009 strategic dictator game}

The 2009 strategic dictator game immediately followed the 2009 dictator game. The strategic dictator game consisted in asking players to guess the amount allocated to each of the dictator game recipients by one of the session's FFF players (although, so as not to prime players to the ethnicity of the FFF model, we did not advertize that this model would specifically be FFF). Players were also told that the player who guessed closest to the actual decisions of this FFF model would receive a prize of 30 euros. Moreover, they were informed that, in case of a tie, all subjects who made the closest guess would receive this prize The strategic dictator game therefore helps us determine the beliefs of our 2009 players about FFF generosity toward the various recipients in the 2009 dictator game.

(being assured that the amounts accruing to each recipient will actually be transferred to them). There is no penalty for keeping the entire amount, and no one can influence the players' donations: they are therefore, effectively, "dictators".

${ }^{3}$ Here is the way the 2009 dictator game was introduced to the subjects: "We are going to play one last game. In this game, we are projecting pictures of individuals on the wall. You will see a total of six pictures, sequentially. For each face, we will give you 5 euros. You are to decide how much of these 5 euros $(0,1,2,3,4$ or all of it, 5$)$ you wish to give to the individual in the picture. Let me emphasize that these individuals whose pictures are projected are real individuals. They were recruited by our teams the same way you were. We will contact them again after the experiment to give them the amount of money that you decided to give them. Keep in mind however that these individuals will never know who you are or how much you will have given them. Your decision is therefore entirely private. Do you have any questions?".

${ }^{4}$ This reward scheme is incentive compatible provided the 10 subjects cannot coordinate on a random value between 0 and 5 . Given that subjects were not allowed to talk to each other, this condition was satisfied. As an illustration, two of our ten subjects made the same closest guess in only 1 of our 8 game sessions. 


\section{Appendix A3: Analysis of FFF guesses in the 2010 strategic dictator game}

In the 2010 strategic dictator game, the 50 FFF players were shown, sequentially, photographs of some of our 2009 players. Underneath the photograph, they were also shown photographs of the six recipients from the 2009 dictator game. FFF players were asked to guess the donation of the pictured 2009 dictator to each of the six recipients. Players received 50 centimes for each correct answer 1 Figure A3 illustrates a sample screenshot of the 2010 strategic dictator game.

Figure A3: Sample screenshot of the 2010 strategic dictator game.

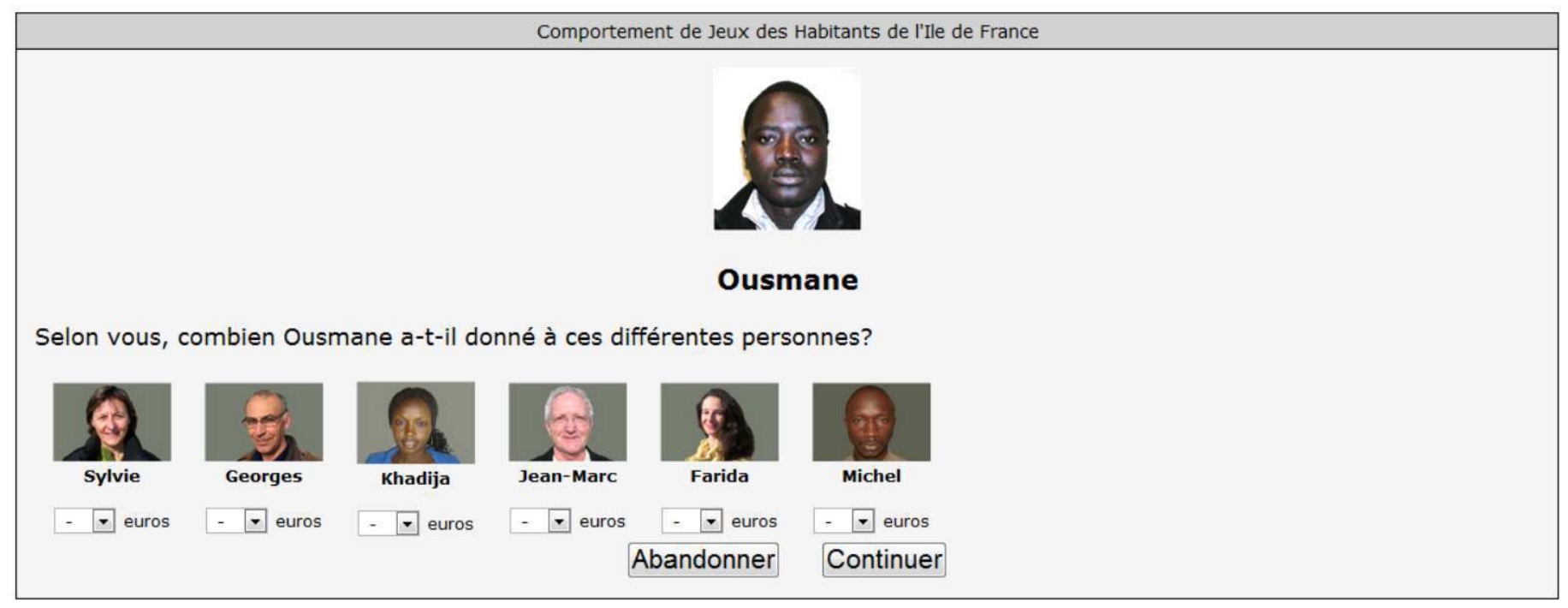

Translation of Figure A3: "How many euros do you think Ousmane gave to these people?"

Two versions of the game were prepared, but only one of them (randomly chosen) was selected for each game session. The same Senegalese dictator was ascribed a Muslim name in one version and a Christian name in the other. This allows us to run a within-dictator analysis (i.e.: to control for the face of the dictator) when we compare FFF guesses about SM dictator donations to FFF recipients with FFF guesses about SX dictator donations to FFF recipients.

This comparison enables us to determine whether FFF expect SM to show lower levels (as compared to SX) of unconditional altruism toward FFF recipients before socialization (our $2010 \mathrm{FFF}$ players were indeed not aware that a speed chatting game had occurred before the 2009 dictator game).

We estimate the following equation over the set of triads composed of FFF guessers, SM and SX dictators and FFF recipients:

$$
y=a+b \cdot(\mathrm{FFF} \rightsquigarrow \mathrm{SM} \rightarrow \mathrm{FFF})+\mathbf{c}^{\prime} \cdot \mathbf{X}+\mathbf{d}^{\prime} \cdot \mathbf{Z}+\mathbf{e}^{\prime} . \mathbf{H}+\mathbf{f}^{\prime} . \mathbf{\Pi}+\epsilon,
$$

where $y$ refers to FFF guesses about SM or SX donation (or unconditional altruism) to FFF recipients. The dummy $(\mathrm{FFF} \rightsquigarrow \mathrm{SM} \rightarrow \mathrm{FFF})$ is equal to 1 if the guesser is FFF, the dictator is $\mathrm{SM}$ and the recipient is FFF and to 0 if the guesser is FFF, the dictator is $\mathrm{SX}$ and the recipient is FFF. As a consequence, coefficient $b$ captures the difference between FFF guesses about SM dictators' donations to FFF recipients and FFF guesses about SX dictators' donations to FFF recipients. We control for a series of socioeconomic characteristics of FFF guessers (gender, age, education, household income and religiosity) that are denoted by $\mathbf{X}$. Additionally, in order to run a within-face analysis, we introduce $\mathbf{Z}$ and $\mathbf{H}$, two vectors of face fixed effects for the dictator and the recipient respectively. Finally, we introduce $\boldsymbol{\Pi}$, a vector of session fixed effects. Note that standard errors are clustered at the guesser level since guesses from the same guesser cannot be considered independent of one another.

Table A3 presents OLS estimates from three model specifications. In column 1, we control for the dummy (FFF $\rightsquigarrow$ SM $\rightarrow$ FFF) only. In column 2, we add dictator- and recipient- face fixed effects as well as session fixed effects. In column 3 , we include the socio-demographic characteristics of the guesser. The non significant coefficient in row (1) in all three columns shows that guesses of the 2010 FFF players about the donation of SM and SX dictators to FFF recipients do not differ.

\footnotetext{
${ }^{5}$ In cases where FFF had to guess donation amounts for player match-ups that never actually occurred in 2009, they were automatically given 25 centimes.
} 
Table A3: Guesses of FFF players in the 2010 strategic dictator game. OLS analysis.

\begin{tabular}{lccc}
\hline \hline & \multicolumn{3}{c}{ Dep. var.: Guesses of FFF players } \\
& $(1)$ & $(2)$ & $(3)$ \\
\hline$(1) \mathrm{FFF} \rightsquigarrow \mathrm{SM} \rightarrow \mathrm{FFF}$ & -0.013 & -0.000 & -0.008 \\
& $(0.068)$ & $(0.064)$ & $(0.066)$ \\
SES of the guesser & & &
\end{tabular}

SES of the guesser

(2) Female

$-0.289$

(3) Age

$(0.184)$

$-0.001$

(4) Household income

(5) Education

$(0.040)$

(6) Religiosity

$0.249^{* * *}$

$(0.065)$

\begin{tabular}{lccc}
\hline \hline Session fixed effects & No & Yes & Yes \\
Dictator face fixed effects & No & Yes & Yes \\
Recipient face fixed effects & No & Yes & Yes \\
\hline $\mathrm{R}^{2}$ & 0.000 & 0.138 & 0.400 \\
Observations & 600 & 600 & 576 \\
\hline \hline
\end{tabular}

Notes: The table reports OLS estimates. The unit of observation is a triad formed by a FFF guesser, a SM or SX dictator and a FFF recipient. The dependent variable ranges from 0 (the guesser guesses that the donor gives nothing to the recipient) to 5 (the guesser guesses that the donor gives her total endowment to the recipient). "FFF $\rightsquigarrow \mathrm{SM} \rightarrow \mathrm{FFF}$ " takes the value 1 if the guesser is $\mathrm{FFF}$, the dictator is $\mathrm{SM}$ and the recipient is FFF and 0 if the guesser is FFF, the dictator is SX and the recipient is FFF. "Female" takes the value 1 if the FFF guesser is male and 0 otherwise. "Age" is equal to the age of the FFF guesser. "Education" ranges from 1 (less than primary school completed) to 10 (higher than college degree completed). "Household income" ranges from 1 (less than 500 euros monthly) to 11 (more than 7,500 euros monthly). "Religiosity" ranges from 1 (never attends religious services) to 7 (attends religious services several times a week). Standard errors are clustered at the FFF guesser level. *,** and $* * *$ indicate significance at the 10,5 and $1 \%$ levels.

\section{Appendix A4: Analysis of FFF guesses in the 2010 double strategic dictator game}

In the 2010 strategic dictator game, the 50 FFF players were shown the photographs of two of our 2009 Senegalese female players, as well as of two of our $2009 \mathrm{FFF}$ female dictators. Underneath these photographs, they were also shown the photographs of the six recipients from the 2009 dictator game. Our $2010 \mathrm{FFF}$ players were asked to guess the guesses of the 2009 Senegalese female players during the 2009 strategic dictator game about the amount that the $2009 \mathrm{FFF}$ dictators had transferred to each of the six recipients from the 2009 dictator game. Players received 50 centimes for each correct answer Figure A4 illustrates a sample screenshot of the 2010 double strategic dictator game.

\footnotetext{
${ }^{6}$ In cases where FFF had to guess donation amounts for player match-ups that never actually occurred in 2009, they were automatically given 25 centimes.
} 
Figure A4: Sample screenshot of the double 2010 strategic dictator game.

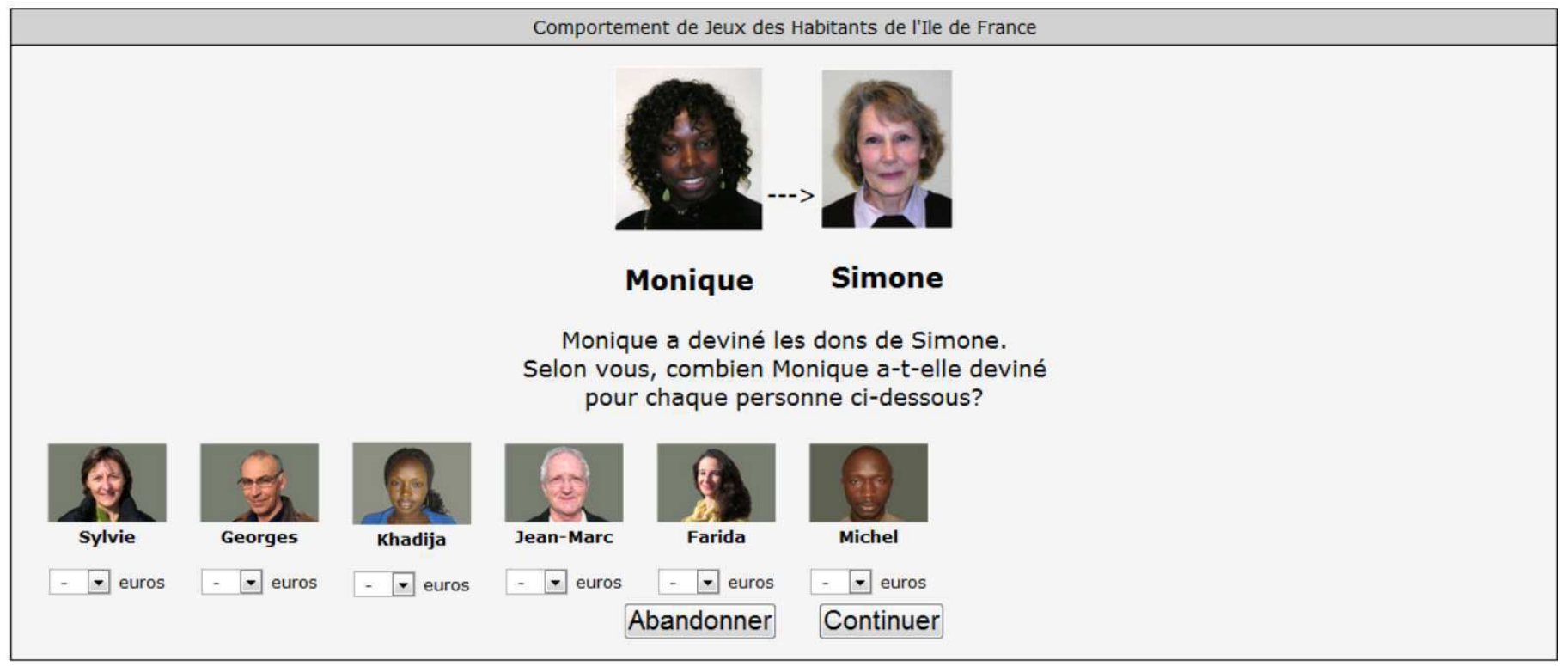

Translation of Figure A4: "Monique has guessed Simone's donations. What do you think is Monique's guess for each of the individuals below?"

Two versions of the game were prepared but only one of them (randomly chosen) was selected for each game session. In order to run a within-Senegalese player analysis (i.e., to control for the face of this player), the same 2009 Senegalese player was ascribed the Muslim first name "Aicha" in one version, and the Christian first name "Monique" in the other.

The 2010 double strategic dictator game allows us to determine whether FFF hold different second-order beliefs about SM and SX, that is whether they expect SM beliefs about FFF unconditional altruism toward SM to be more pessimistic than SX beliefs about FFF unconditional altruism toward SX.

We estimate the following equation over the set of quatuors composed of a FFF guesser, a SM or SX player, a FFF dictator and a SM or SX recipient:

$$
y=a+b .(\mathrm{FFF} \rightsquigarrow \mathrm{SM} \rightsquigarrow \mathrm{FFF} \rightarrow \mathrm{SM})+\mathbf{c}^{\prime} \cdot \mathbf{X}+\mathbf{d}^{\prime} \cdot \mathbf{Z}+\mathbf{e}^{\prime} \cdot \mathbf{H}+\mathbf{f}^{\prime} \cdot \mathbf{J}+\mathbf{g}^{\prime} \cdot \mathbf{\Pi}+\epsilon,
$$

where $y$ refers to FFF guesses on SM or SX players' guesses on FFF dictators' donations to SM or SX recipients. The dummy (FFF $\rightsquigarrow \mathrm{SM} \rightsquigarrow \mathrm{FFF} \rightarrow \mathrm{SM}$ ) is equal to 1 if the guesser is FFF, the player is SM, the dictator is FFF and the recipient is SM and 0 if the guesser is FFF, the player is SX, the dictator is FFF and the recipient is SX. As a consequence, coefficient $b$ captures the difference between FFF guesses about SM guesses on FFF dictators' donations to SM recipients and FFF guesses about SX guesses on FFF dictators' donations to SX recipients. We control for a series of socioeconomic characteristics of FFF guessers (gender, age, education, household income and religiosity) that are denoted by $\mathbf{X}$. Additionally, in order to run a within-face analysis, we introduce $\mathbf{Z}, \mathbf{H}$ and $\mathbf{J}$, three vectors of face fixed effects for the player, the dictator and the recipient respectively. Finally, we introduce $\boldsymbol{\Pi}$, a vector of session fixed effects. Note that standard errors are clustered at the guesser level since guesses from the same guesser cannot be considered independent of one another.

Table A4 presents OLS estimates from three model specifications. In column 1, we control for the dummy (FFF $\rightsquigarrow \mathrm{SM} \rightsquigarrow$ FFF $\rightarrow$ SM) only. In column 2, we add player-, dictator- and recipient- face fixed effects as well as session fixed effects. In column 3, we include the socio-demographic characteristics of the guesser. The non significant coefficient in row (1) in all three columns shows that guesses of the 2010 FFF players about SM guesses on FFF dictators' donations to SM recipients are similar to their guesses about SX guesses on FFF dictators' donations to SX recipients. 
Table A4: Guesses of FFF players in the 2010 double strategic dictator game. OLS analysis.

\begin{tabular}{|c|c|c|c|}
\hline & \multicolumn{3}{|c|}{$\begin{array}{l}\text { Dep. var.: Guesses of FFF players } \\
(1)\end{array}$} \\
\hline (1) $\mathrm{FFF} \rightsquigarrow \mathrm{SM} \rightsquigarrow \mathrm{FFF} \rightarrow \mathrm{SM}$ & $\begin{array}{c}0.020 \\
(0.132)\end{array}$ & $\begin{array}{l}-0.002 \\
(0.128)\end{array}$ & $\begin{array}{c}0.027 \\
(0.133)\end{array}$ \\
\hline SES of the guesser & & & \\
\hline (2) Female & & & $\begin{array}{c}0.074 \\
(0.267)\end{array}$ \\
\hline (3) Age & & & $\begin{array}{c}0.006 \\
(0.011)\end{array}$ \\
\hline (4) Household income & & & $\begin{array}{c}0.006 \\
(0.055)\end{array}$ \\
\hline (5) Education & & & $\begin{array}{c}0.179^{* * * *} \\
(0.057)\end{array}$ \\
\hline (6) Religiosity & & & $\begin{array}{c}0.287^{* * * *} \\
(0.089)\end{array}$ \\
\hline Session fixed effects & $\overline{\mathrm{No}}$ & Yes & Yes \\
\hline Senegalese player face fixed effects & No & Yes & Yes \\
\hline FFF dictator face fixed effects & No & Yes & Yes \\
\hline Recipient face fixed effects & No & Yes & Yes \\
\hline $\mathrm{R}^{2}$ & 0.000 & 0.177 & 0.374 \\
\hline Observations & 200 & 200 & 192 \\
\hline
\end{tabular}

Notes: The table reports OLS estimates. The unit of observation is a quatuor formed by a FFF guesser, a SM or SX player, a FFF dictator and a SM or SX recipient. The dependent variable ranges from 0 (the guesser guesses that the player guesses that the FFF dictator gives nothing to the recipient) to 5 (the guesser guesses that the player guesses that the FFF dictator gives her total endowment to the recipient). "FFF $\rightsquigarrow \mathrm{SM}$ $\rightsquigarrow \mathrm{FFF} \rightarrow \mathrm{SM}$ " takes the value 1 if the guesser is FFF, the player is SM, the dictator is $\mathrm{FFF}$ and the recipient is $\mathrm{SM}$ and 0 if the guesser is $\mathrm{FFF}$, the player is $\mathrm{SX}$, the dictator is FFF and the recipient is SX. "Female" takes the value 1 if the FFF guesser is male and 0 otherwise. "Age" is equal to the age of the FFF guesser. "Education" ranges from 1 (less than primary school completed) to 10 (higher than college degree completed). "Household income" ranges from 1 (less than 500 euros monthly) to 11 (more than 7,500 euros monthly). "Religiosity" ranges from 1 (never attends religious services) to 7 (attends religious services several times a week). Standard errors are clustered at the FFF guesser level. $*$, ** and $* * *$ indicate significance at the 10,5 and $1 \%$ levels.

\section{Appendix A5: Analysis of FFF guesses in the 2010 incomes game}

In this game, we showed our $2010 \mathrm{FFF}$ players the pictures of the six recipients from the 2009 dictator game with their ascribed first names and asked them to guess the monthly income of each of these individuals. Half of the FFF players saw the picture of the Senegalese female recipient and the picture of the Senegalese male recipient with the ascribed first names "Khadija" and "Michel" respectively, and half saw the picture of the Senegalese female recipient and the picture of the Senegalese male recipient with the ascribed first names "Joséphine" and "Aboubacar" respectively. This experimental set up allows us to test whether, holding the picture of the Senegalese constant, FFF hold different beliefs about SM versus SX monthly income.

We estimate the following equation over the set of pairs composed of a FFF guesser and a SM or SX recipient:

$$
y=a+b \cdot(\mathrm{FFF} \rightsquigarrow \mathrm{SM})+\mathbf{c}^{\prime} \cdot \mathbf{X}+\mathbf{d}^{\prime} \cdot \mathbf{Z}+\mathbf{e}^{\prime} \cdot \mathbf{\Pi}+\epsilon,
$$

where $y$ refers to FFF guesses about SM or SX monthly income. The dummy (FFF $\rightsquigarrow \mathrm{SM}$ ) is equal to 1 if the recipient is $\mathrm{SM}$ and to 0 if the recipient is SX. As a consequence, coefficient $b$ captures the difference between FFF guesses about SM monthly income and FFF guesses about SX monthly income. We control for a series of socioeconomic characteristics of FFF guessers (gender, age, education, household income and religiosity) that are denoted by $\mathbf{X}$. Additionally, in order to run a within-face analysis, we introduce $\mathbf{Z}$, a vector of face fixed effects for the recipient. Finally, we introduce $\boldsymbol{\Pi}$, a vector of session fixed effects. Note that standard errors are clustered at the guesser level. As a matter of fact, guesses stemming from the same guesser cannot be considered independent of one another.

Table A5 presents OLS estimates from three model specifications. In column 1, we control for the dummy (FFF $\rightsquigarrow \mathrm{SM}$ ) only. In column 2 , we add recipient face fixed effects as well as session fixed effects. In column 3, we include the sociodemographic characteristics of the guesser. The non significant coefficient in row (1) in all three columns shows that guesses of the $2010 \mathrm{FFF}$ players about the monthly income of SM and SX recipients do not differ. 
Table A5: FFF beliefs about SM and SX monthly income. OLS analysis.

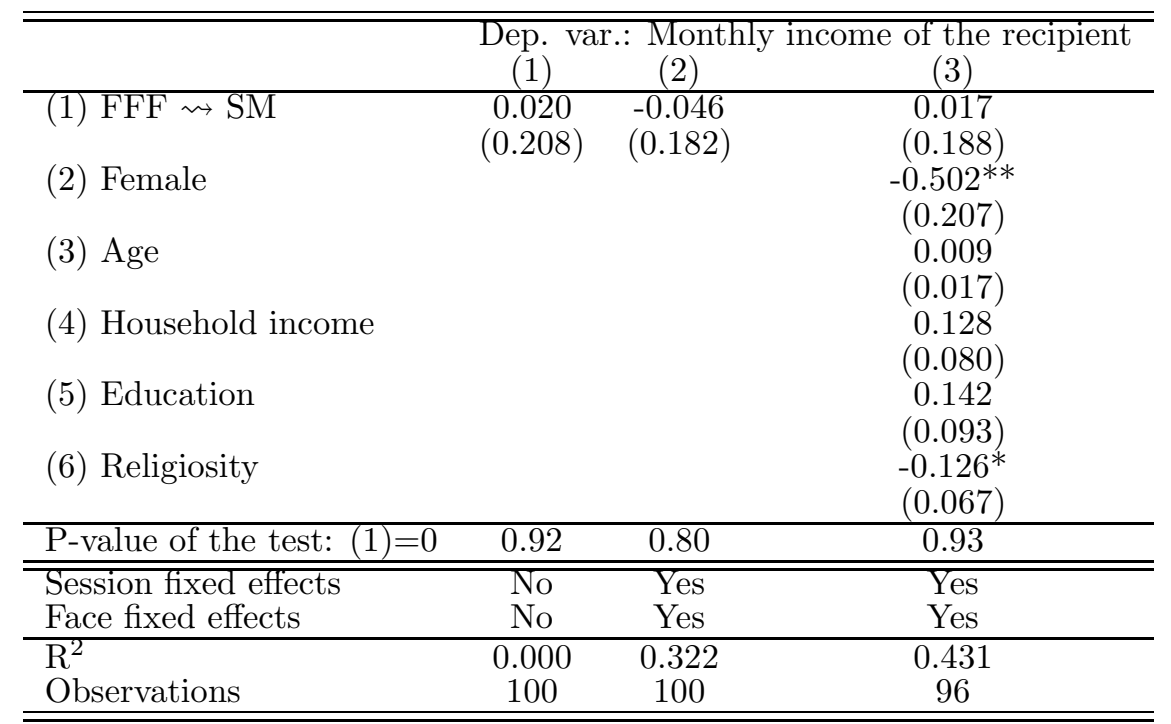

Notes: The table reports OLS estimates. The unit of observation is a dyad formed of FFF guessers and SM or SX recipients. The dependent variable ranges from 1 (less than 500 euros monthly) to 11 (more than 7,500 euros monthly). "FFF $\rightsquigarrow \mathrm{SM}$ " takes the value 1 if the recipient is SM, and 0 if the recipient is SX. "Female" takes the value 1 if the FFF player is female and 0 otherwise. "Age" is equal to the age of the FFF player. "Education" ranges from 1 (less than primary school completed) to 10 (higher than college degree completed). "Household income" ranges from 1 (less than 500 euros monthly) to 11 (more than 7,500 euros monthly). "Religiosity" ranges from 1 (never attends religious services) to 7 (attends religious services several times a week). Standard errors are clustered at the FFF guesser level. *, ** and *** indicate significance at the 10, 5 and $1 \%$ levels. 